\title{
Slow energy dissipation in anharmonic oscillator chains
}

December 21, 2007

\author{
Martin Hairer ${ }^{1}$ and Jonathan C. Mattingly ${ }^{2}$ \\ 1 Mathematics Institute, The University of Warwick, Coventry CV4 7AL, UK \\ Email: M.Hairer@Warwick.ac.uk \\ 2 Department of Mathematics and Center for Nonlinear and Complex Systems, \\ Duke University, Durham 27701, USA \\ Email: jonm@math.duke.edu
}

\begin{abstract}
We study the dynamic behavior at high energies of a chain of anharmonic oscillators coupled at its ends to heat baths at possibly different temperatures. In our setup, each oscillator is subject to a homogeneous anharmonic pinning potential $V_{1}\left(q_{i}\right)=\left|q_{i}\right|^{2 k} / 2 k$ and harmonic coupling potentials $V_{2}\left(q_{i}-q_{i-1}\right)=\left(q_{i}-q_{i-1}\right)^{2} / 2$ between itself and its nearest neighbors. We consider the case $k>1$ when the pinning potential is stronger then the coupling potential. At high energy, when a large fraction of the energy is located in the bulk of the chain, breathers appear and block the transport of energy through the system, thus slowing its convergence to equilibrium.

In such a regime, we obtain equations for an effective dynamics by averaging out the fast oscillation of the breather. Using this representation and related ideas, we can prove a number of results. When the chain is of length three and $k>3 / 2$ we show that there exists a unique invariant measure. If $k>2$ we further show that the system does not relax exponentially fast to this equilibrium by demonstrating that zero is in the essential spectrum of the generator of the dynamics. When the chain has five or more oscillators and $k>3 / 2$ we show that the generator again has zero in its essential spectrum.

In addition to these rigorous results, a theory is given for the rate of decrease of the energy when it is concentrated in one of the oscillators without dissipation. Numerical simulations are included which confirm the theory.
\end{abstract}

\section{Introduction}

One subject that has received considerable attention in recent years is the return to equilibrium of systems arising from statistical mechanics. One of the simplest models of interest is given by the kinetic Fokker-Planck equation

$$
\partial_{t} \varphi_{t}=\mathcal{L} \varphi_{t}, \quad \varphi_{0}=\varphi, \quad \mathcal{L}=\frac{1}{2} \partial_{p}^{2}-p \partial_{p}+p \partial_{q}-\nabla V(q) \partial_{p}, \quad p, q \in \mathbf{R}^{n}
$$

This equation describes the evolution of an observable $\varphi: \mathbf{R}^{n} \rightarrow \mathbf{R}$ under the Hamiltonian dynamic for the energy $H(p, q)=\frac{p^{2}}{2}+V(q)$, perturbed by friction and noise:

$$
d p=-\nabla V(q) d t-p d t+d w(t), \quad d q=p d t .
$$


The relation between (1.2) and (1.1) is given by the fact that the function $\varphi_{t}\left(p_{0}, q_{0}\right)=$ $\mathbf{E} \varphi_{0}(p(t), q(t))$ satisfies the partial differential equation (1.1), provided that the pair $(p(t), q(t))$ solves (1.2). It can easily be checked by inspection that if $V$ is sufficiently smooth and coercive, the measure $\mu=\exp (-2 H(p, q)) d p d q$ is invariant under this dynamic in the sense that if $\varphi_{t}$ satisfies (1.1), then $\int \varphi_{t} d \mu$ does not depend on $t$. This can be rephrased as saying that if $\left(p_{0}, q_{0}\right)$ is a random variable with law $\mu$ independent of the driving noise $w$, then the law of $(p(t), q(t))$ is given by $\mu$ for all times.

Underpinning much of the analysis of $\mathcal{L}$ on the space $\mathrm{L}^{2}(\mu)$ is the guiding principle that it related to the corresponding Witten Laplacian [HN05]

$$
\Delta_{V}=-\Delta_{q}+|\nabla V(q)|^{2}-(\Delta V)(q)
$$

In particular, it was conjectured by Helffer and Nier [HN05, Conjecture 1.2] that $\mathcal{L}$ has compact resolvent on $L^{2}(\mu)$ if and only if $\Delta_{V}$ has compact resolvent on the flat space $\mathrm{L}^{2}\left(\mathbf{R}^{n}\right)$. This conjecture has been partially solved in [HN05] (see also [Nie06]) in the sense that one can exhibit a large class of potentials for which it holds (loosely, $V$ should grow in a sufficiently regular way at infinity). Recently, upper as well as lower bounds on the spectral gap of $\mathcal{L}$ have been obtained in [HN04, Vil07] for potentials $V$ that satisfy certain growth conditions at infinity.

All of the results cited above make heavy use of the following two key facts:

a. There is an explicit formula for the invariant measure of (1.2).

b. The friction term $-p \partial_{p}$ acts on all (physical) degrees of freedom of the system.

Both of these facts are violated in the following very popular model for heat conduction. Take a finite collection of $N+1$ anharmonic oscillators with nearest-neighbor couplings, that is a classical Hamiltonian system with Hamiltonian given by

$$
H(p, q)=\sum_{i=0}^{N}\left(\frac{p_{i}^{2}}{2}+V_{1}\left(q_{i}\right)\right)+\sum_{i=1}^{N} V_{2}\left(q_{i}-q_{i-1}\right) .
$$

Here, the potential $V_{2}$ is the interaction potential between neighboring oscillators, whereas $V_{1}$ is a pinning potential. This system is then put in contact with two heat baths at different temperatures $T_{0}$ and $T_{N}$. We model the interaction with the heat baths by the standard Langevin dynamics, so that the equations of motion of our system are given by

$$
\begin{aligned}
d p_{0} & =-\gamma_{0} p_{0} d t-V_{1}^{\prime}\left(q_{0}\right) d t-V_{2}^{\prime}\left(q_{0}-q_{1}\right) d t+\sigma_{0} d w_{0} \\
d p_{i} & =-V_{1}^{\prime}\left(q_{i}\right) d t-V_{2}^{\prime}\left(q_{i}-q_{i-1}\right) d t-V_{2}^{\prime}\left(q_{i}-q_{i+1}\right) d t \\
d p_{N} & =-\gamma_{N} p_{N} d t-V_{1}^{\prime}\left(q_{N}\right) d t-V_{2}^{\prime}\left(q_{N}-q_{N-1}\right) d t+\sigma_{N} d w_{N} \\
d q_{j} & =p_{j} d t .
\end{aligned}
$$

Here, we set $\sigma_{i}^{2}=2 \gamma_{i} T_{i}$, the index $i$ runs from 1 to $N-1$ and the index $j$ runs from 0 to $N$. As described in [BLR00], the rigorous analysis of this model and in particular the derivation of Fourier's law (or the proof of its breakdown) is an outstanding mathematical problem of great interest to the applied community.

If $T_{0}=T_{N}=T$, then one can check as before that this set of equations has a unique invariant measure, which has density $\exp (-H(p, q) / T)$ with respect to the Lebesgue measure, where $H$ is the Hamiltonian from (1.4). When the two temperatures $T_{0}$ and $T_{N}$ are different however, much less is known. In particular, as we will see immediately, even the existence of an invariant probability measure is an open problem in some cases as simple as $V_{1}(q)=q^{4}$ and $V_{2}(q)=q^{2}$. 
This model has been the subject of many studies, both from a numerical and from a theoretical perspective. The purely harmonic case has been solved explicitly in [RLL67]. Though no explicit solution is known in the anharmonic case, a wealth of numerical experiments exist, see for example [LLP03] and references therein. Since we will mainly focus on the theoretical aspects of the model, we refer to [EPR99a, EPR99b], which seems to be one of the first rigorous studies of the anharmonic case. It was shown in [EH00, EH03] that if $V_{1}(q)$ and $V_{2}(q)$ behave approximately like $|q|^{a_{1}}$ and $|q|^{a_{2}}$ respectively at infinity then, provided that $a_{2}>a_{1}>2$, there exists a unique invariant measure for (1.5). The statement that was proved was actually stronger than that, namely it was shown that the generator $\mathcal{L}$ of (1.5) has compact resolvent in every space of the form $L^{2}(\exp (-H(p, q) / T) d p d q)$ with $T>\max \left\{T_{0}, T_{N}\right\} / 2$. In [RBT02], it was also shown, using entirely probabilistic rather than functional-analytic techniques, that the condition $a_{2} \geq a_{1} \geq 2$ is sufficient for the existence and uniqueness of an invariant measure for (1.5). Furthermore, the compactness of the corresponding semigroup in some weighted $L^{\infty}$ space was also proved there.

This left open the case $a_{2}<a_{1}$ which is the subject of the present study. To our knowledge, no previous rigorous results exist in this case, although some interesting theory has been developed recently in [BK07, LS05]. At first sight, one might think that there is no a priori reason for the behavior of (1.5) to differ in any essential way from the case $a_{2} \geq a_{1}$ where spectral gap results are known. Such wishful thinking turns out to be overly optimistic. Even in the simplest possible scenario, that is when $V_{1}(q)=q^{4}$ and $V_{2}(q)=q^{2}$, we will show in Theorem 3.11 below that the compactness property of the resolvent of $\mathcal{L}$ is destroyed as soon as $N+1 \geq 3$. Furthermore, when $N+1 \geq 5$, it will be shown in Theorem 3.13 that the essential spectrum of $\mathcal{L}$ (always in a weighted $L^{2}$ space of the type considered before) extends all the way to 0 . These negative results hold even in the case where $T_{0}=T_{N}=T$, showing that having the friction acting on all physical degrees of freedom is a crucial assumption for the Helffer-Nier conjecture to hold.

The reason why the behavior of (1.5) changes so drastically when $a_{2}<a_{1}$ can be understood heuristically by the appearance of breathers (see for example [MA94]). Breathers are dynamically stable, spatially localized, periodic orbits that arise in the noise-free translation invariant $(i=-\infty, \ldots,+\infty)$ version of (1.5). Good approximations to these orbits persist in (1.5), especially if $N$ is large. It is therefore possible to put the system into a state where most of its energy is localized in a few oscillators located in the middle of the chain. On the other hand, energy can be dissipated only through the terms $-\gamma p$ appearing in the equations for the first and the last oscillator. Therefore, one expects the energy of the system to decay extremely slowly. The appearance of breathers can be proved in the case where the strength of the nearest-neighbor coupling is much weaker than the strength of the pinning potential. At high energies, this is precisely the case when $a_{2}<a_{1}$.

This discussion shows that even the mere existence of an invariant measure is a nontrivial problem in this model unless $T_{0}=T_{N}$, in which case one can check explicitly that the usual Boltzmann-Gibbs distribution is invariant. This differs from many other systems in that the existence of an invariant measure, not its uniqueness, is difficult. In fact, the uniqueness of an invariant measure for a chain of arbitrary length follows quickly from the hypoellipticity of the generator and the Hamiltonian structure once the existence of an invariant measure is established.

We are at the moment unable to provide a general proof that shows the existence of an invariant measure for a chain of arbitrary length. However, in the case of a chain comprising of 3 oscillators, we show in Theorem 5.6 below that there exists a unique 
invariant measure, provided that the coupling potential is harmonic and the pinning potential is homogeneous of sufficiently high degree. This result is proven by first obtaining an effective dynamics when most of the energy is concentrated in the central oscillator. This effective dynamics is then used to construct a Lyapunov function whose level sets are compact and hence, by a variation on the classical Kryloff-Bogoliouboff method, implies the existence of an invariant measure.

The remainder of this article is organized as follows. In Section 2, we give a formal calculation that shows how it is possible to relate the spectral properties of $\mathcal{L}$ to the scaling properties of the potentials $V_{1}$ and $V_{2}$. The results given by these formal calculations are then compared to numerical simulations. We proceed in Section 3 to the proof of the negative results concerning the lack of compactness and/or of a spectral gap for $\mathcal{L}$. In Section 4 we derive effective equations of motion for the system of three oscillators in the regime when a breather is present. Here, we make heavy use of the compensator techniques from averaging / homogenization theory [BLP78]. Finally, we show in Section 5 that in the simplest case of a chain of three oscillators with harmonic coupling potentials, one can show the existence of an invariant measure for any degree of homogeneity greater than 2 for the pinning potential. Perhaps more interesting then this last result is the method of proof. We derive as system of effective equations and prove their accuracy that when the energy of middle oscillator is large. These effective equations give insight into the mechanism of energy dissipation and are used to construct a Lyapunov function.

\section{Acknowledgements}

We would like to thank the University of Bonn (and the then-nascent Hausdorff institute) for providing the pleasant working environment where this project began. $\mathrm{MH}$ would like to thank Jean-Pierre Eckmann and Luc Rey-Bellet for many long discussions about this problem. We would also like to thank Arnaud Guillin for his encouragements which aided in completing this text in a (more or less) timely manner. MH acknowledges support from EPSRC fellowship EP/D071593/1. JCM acknowledges the support of the Sloan Foundation and National Science Foundation through a PECASE award (DMS 04-49910) and a standard award (DMS 06-16710).

\section{A formal calculation}

In this section, we first perform a formal calculation that allows us to get a feeling of the speed at which energy is extracted from such a system. Consider the simplest nontrivial case, that is when $N+1=3$. In order to keep things simple, we furthermore assume that the coupling potential $V_{2}$ is quadratic: $V_{2}(q)=q^{2} / 2$ and that the pinning potential is homogeneous of degree $2 k: V_{1}(q)=|q|^{2 k} /(2 k)$ for some real number $k>1$. The equations of motion for the system of interest are thus given by

$$
\begin{aligned}
& d p_{0}=-\gamma_{0} p_{0} d t-q_{0}\left|q_{0}\right|^{2 k-2} d t-\left(q_{0}-q_{1}\right) d t+\sqrt{2 \gamma_{0} T_{0}} d w_{0} \\
& d p_{1}=-q_{1}\left|q_{1}\right|^{2 k-2} d t-\left(2 q_{1}-q_{0}-q_{2}\right) d t \\
& d p_{2}=-\gamma_{2} p_{2} d t-q_{2}\left|q_{2}\right|^{2 k-2} d t-\left(q_{2}-q_{1}\right) d t+\sqrt{2 \gamma_{2} T_{2}} d w_{2} \\
& d q_{j}=p_{j} d t .
\end{aligned}
$$

Let us first have a look at the motion of the middle oscillator by itself, i.e. at the solution of

$$
d p=-q|q|^{2 k-2} d t, \quad d q=p d t .
$$


It is easy to see that this equation is invariant under the substitution

$$
q(t)=E^{\frac{1}{2 k}} \tilde{q}\left(E^{\alpha} t\right), \quad p(t)=E^{\frac{1}{2}} \tilde{p}\left(E^{\alpha} t\right),
$$

with $\alpha=\frac{1}{2}-\frac{1}{2 k}$. Let us now denote by $(\tilde{p}, \tilde{q})$ the solution to (2.2) with initial condition $(\sqrt{2}, 0)$, so that $(p, q)$ as given by (2.3) is the (unique up to a phase) solution to (2.2) at energy $E$. Since the variables $p$ and $q$ are assumed to be one-dimensional, the solution $(\tilde{p}, \tilde{q})$ is periodic, say with period $\tau$.

Consider now the equation for the left oscillator, into which we substitute the (approximate) solution to the motion of the middle oscillator that we just found:

$$
\begin{aligned}
& d p_{0}=-\gamma_{0} p_{0} d t-q_{0}\left|q_{0}\right|^{2 k-2} d t+q_{0} d t+\sqrt{2 \gamma_{0} T_{0}} d w_{0}+E^{\frac{1}{2 k}} \tilde{q}\left(E^{\alpha} t\right) d t \\
& d q_{0}=p_{0} d t .
\end{aligned}
$$

If $E$ is large compared to the typical size of $\left(p_{0}, q_{0}\right)$ we expect that, up to lower order corrections, the solution to this equation behaves like the superposition of the solution $\left(\bar{p}_{0}, \bar{q}_{0}\right)$ to $(2.4)$ with the exogenous forcing $\tilde{q} \equiv 0$ and of a highly oscillatory term of the form

$$
\tilde{p}_{0}=E^{\frac{1}{2 k}-\alpha} P\left(E^{\alpha} t\right), \quad \dot{P}=\tilde{q} .
$$

By symmetry, the same applies to the right oscillator $\left(p_{2}, q_{2}\right)$. Applying now Itô's formula to the total Hamiltonian $H$ for (2.1) yields

$$
\frac{d}{d t} \mathbf{E} H(t)=\mathbf{E}\left(\gamma_{0} T_{0}+\gamma_{2} T_{2}-\gamma_{0} p_{0}^{2}(t)-\gamma_{2} p_{2}^{2}(t)\right)
$$

In light of the above discussion, we take $\left(p_{i}, q_{i}\right) \approx\left(\bar{p}_{i}+\tilde{p}_{i}, \bar{q}_{i}+\tilde{q}_{i}\right)$ for $i=0,2$ so $p_{i}^{2}=\bar{p}_{i}^{2}+2 \bar{p}_{i} \tilde{p}_{i}+\tilde{p}_{i}^{2}$. From its definition, observe that $\mathbf{E} \bar{p}_{i}^{2}(t) \rightarrow T_{i}$ as $t \rightarrow \infty$ for $i=0,2$. Furthermore, when the energy $E$ of the middle oscillator is large, we expect the product $\bar{p}_{0}(t) \tilde{p}_{0}(t)$ to average out to a small quantity when integrated over time periods much larger than $E^{-\alpha}$ due to the highly oscillating, mean zero $\tilde{p}_{0}(t)$. Applying this line of reasoning to (2.6) shows that, in the regime where most of the energy of the system is concentrated in the middle oscillator, one expects to have

$$
\frac{d}{d t} H(t) \approx-\left(\gamma_{0} \mathbf{E} \tilde{p}_{0}^{2}+\gamma_{2} \mathbf{E} \tilde{p}_{2}^{2}\right) \approx-\left(\gamma_{0}+\gamma_{2}\right) \kappa_{k} H(t)^{\frac{2}{k}-1},
$$

where $\kappa_{k}$ is the variance of the function $P$ introduced in (2.5), that is

$$
\kappa_{k}=\frac{1}{\tau} \int_{0}^{\tau} P^{2}(s) d s-\frac{1}{\tau^{2}}\left(\int_{0}^{\tau} P(s) d s\right)^{2}, \quad \dot{P}(t)=\tilde{q}(t) .
$$

The dependence on $k$ comes from the fact that $\tilde{q}$ is the position of the free oscillator with potential $\frac{|q|^{2 k}}{2 k}$. Actually, one expects this behavior to be correct even in a chain with more than just three oscillators. If the chain has $N+1=2 n+1$ oscillators and most of the energy is stored in the middle oscillator, one expects the motion of the endpoints to be given by the superposition of a slow motion and a highly oscillatory fast motion $\tilde{p}_{0}$ with a scaling of the type

$$
\tilde{p}_{0}=E^{\frac{1}{2 k}-(2 n-1) \alpha} \hat{P}\left(E^{\alpha} t\right),
$$

where $\hat{P}$ is the (unique) periodic function such that $\frac{d^{2 n-1} \hat{P}}{d t^{2 n-1}}=\tilde{q}$ and such that the integral of $\hat{P}$ over one period vanishes. (This is because we assume that the nearestneighbor couplings are linear.) This suggests that in the general case of a chain of 
length $N+1=2 n+1$, the energy of the system decreases like

$$
\frac{d}{d t} H(t) \approx-\left(\gamma_{0}+\gamma_{N}\right) \kappa_{k, n} H(t)^{\frac{2 n}{k}+1-2 n},
$$

for $\kappa_{k, n}$ the variance of $\hat{P}$ (of course, $\kappa_{k, 1}=\kappa_{k}$ as defined above). If $N+1=2 n$ is even, the worst-case scenario is obtained by storing most of the energy in one of the two middle oscillators. Since their distance to the boundary is the same as the distance of the middle oscillator to the boundary in the chain of length $2 n+1$, we expect the rate of decay of the energy to be similar in both cases.

Remark 2.1 If the coupling potential is not quadratic but homogeneous of degree $2 \ell$, one can still perform a calculation similar to the one we just did, but one has to be more careful. When looking at the influence of $q_{i}$ on $p_{i-1}$ say, one should take into account whether the fast oscillations of $q_{i}$ are of order smaller or larger than 1 . If they are of order smaller than one, one can linearize the coupling potential. If they are or order larger than one, one should multiply them by the scaling exponent arising in the coupling. Suppose as before that the chain contains $N+1=2 n+1$ oscillators and that most of it's energy is stored in the middle oscillator (oscillator $n$ ). Assume that the amplitudes in the fast oscillations of $p_{i}$ and $q_{i}$ scale like $E^{\beta_{i}}$ and $E^{\beta_{i}-\alpha}$ respectively. (Recall that $\alpha=\frac{1}{2}-\frac{1}{2 k}$ is the exponent giving the period of the oscillations.)

One then has $\beta_{n}=1 / 2$. The values of $\beta_{i}$ with $i<n$ are given by the following recursion formula:

$$
\beta_{i}=\left\{\begin{aligned}
(2 \ell-1)\left(\beta_{i+1}-\alpha\right)-\alpha & \text { if } \beta_{i+1}>\alpha \\
\beta_{i+1}-2 \alpha & \text { if } \beta_{i+1} \leq \alpha
\end{aligned}\right.
$$

Using this formula, one can then compute $\gamma=2 \beta_{0}$. Note that if $\ell=1$, one obtains $\beta_{0}=\frac{1}{2}-2 n \alpha$ which agrees with the value for $\gamma$ obtained previously.

Remark 2.2 One would expect these scaling relations to hold at high energies, even if the potentials are not exactly homogeneous. One can then still perform most of the analysis presented below by splitting the right-hand side of the equations into a homogeneous part and a remainder term and by assuming that the remainder gets small (in a suitable sense) at high energies.

\subsection{Numerical simulations}

In this section, we show that there is a surprisingly good agreement, even over extremely long time intervals, between numerical simulations of (2.1) and the predictions (2.7) and (2.9). In order to compare the two, we introduce the function

$$
H^{(k, n)}(p, q)=H^{\beta}(p, q), \quad \beta=2 n\left(1-\frac{1}{k}\right) .
$$

Plugging this into (2.9), we get the prediction

$$
H^{(k, n)}(t) \approx H^{(k, n)}(0)-\left(\gamma_{0}+\gamma_{2}\right) n\left(2-\frac{2}{k}\right) \kappa_{k, n}
$$

A straightforward numerical simulation (essentially integration of the free equation) furthermore allows to compute the values of $\kappa_{k, n}$ to very high precision. For example, we obtain

$$
\kappa_{2,1} \approx 0.63546991, \quad \kappa_{3,1} \approx 0.42363371
$$


We performed numerical simulations for the cases $k=2$ and $k=3$. Both simulations were performed using a modification of the classical Störmer-Verlet method (see for example [HLW06]) to take into account for the friction and the noise. The modification was done in such a way that the resulting method is still of order two.

The simulation for $k=2$ was performed at a stepsize $h=10^{-3}$, and the simulation for $k=3$ was performed at a stepsize $h=4 \cdot 10^{-4}$. Both simulations used $\gamma_{0}=\gamma_{2}=$ 1.3 and $T_{0}=T_{2}=1$.
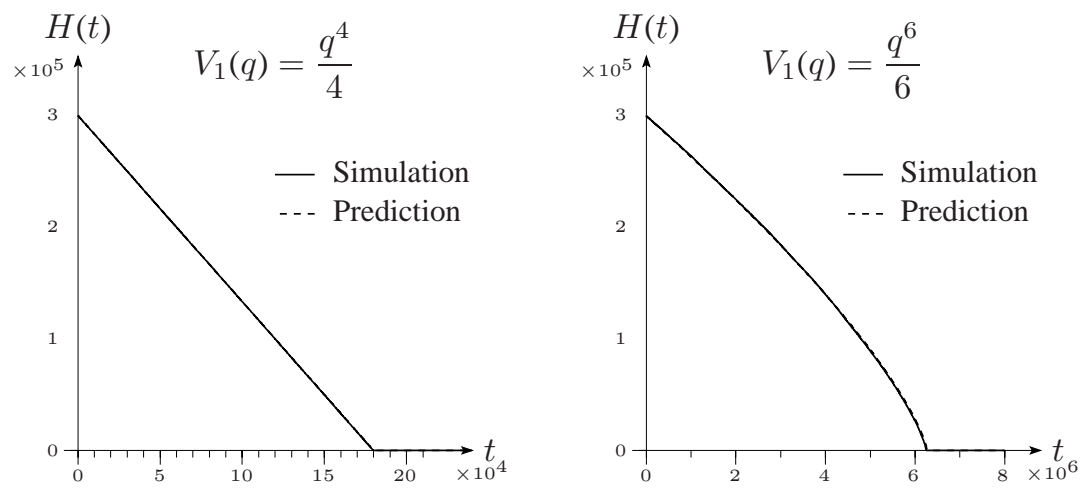

The prediction obtained from (2.10) with the values (2.11) is shown as a dashed line on these figures, but it fits the numerics so well that it is nearly invisible. We emphasize that there were no free parameters in the fit, all constants are predicted by the theory. Note that the timescale in the second picture differs by a factor 40 from the timescale in the first picture.

\subsection{Comparison to a gradient diffusion}

In this section, we argue that the energy decay rate predicted by (2.9) also yields a prediction on the qualitative nature of the spectrum of the generator of (2.1) in the weighted space $L^{2}(\mu)$, where $\mu$ is the invariant measure.

The idea is to model the behavior of the energy $H(t)$ by a one-dimensional diffusion of the type

$$
d x=\left(b(x)+a^{\prime}(x) a(x)\right) d t+a(x) d w(t) .
$$

It is well-known that the invariant measure for (2.12) is given by

$$
\mu(d x)=Z^{-1} \exp \left(2 \int_{0}^{x} \frac{b(t)}{a^{2}(t)} d t\right) .
$$

Since we expect the invariant measure of (2.1) to behave roughly like $\exp (-\beta H) d H$ (up to lower-order corrections), we should choose $a$ such that $a^{2}(x) \approx|b(x)|$ for large $x$. Combining this with (2.9), we obtain the model

$$
b(x)=-x^{\gamma}, \quad a(x)=x^{\gamma / 2}, \quad \gamma=\frac{2 n}{k}+1-2 n,
$$

which has $2 \exp (-2 x) d x$ as its invariant measure (we restrict ourselves to the halfspace $x \geq 0$ ). Note that since $k>1$ (the pinning potential grows faster than the coupling potential), one has always $\gamma<1$.

With the choice (2.13), the generator for (2.12) is then given by

$$
(\mathcal{L} f)(x)=\frac{1}{2} \partial_{x}\left(x^{\gamma} \partial_{x} f\right)(x)-x^{\gamma} \partial_{x} f(x) .
$$


Since the operator $(K f)(x)=f(x) \exp (-x)$ is a unitary operator from the weighted space $L^{2}(\exp (-2 x) d x)$ to the flat $L^{2}$ space, the operator $\mathcal{L}$ is unitarily equivalent to the operator $\mathcal{L}_{1}$ on $L^{2}$ given by

$$
\left(\mathcal{L}_{1} f\right)(x)=\frac{1}{2} \partial_{x}\left(x^{\gamma} \partial_{x} f\right)(x)-\frac{x^{\gamma-1}}{2}(x-\gamma) .
$$

At this point, we recall that if $\varphi: \mathbf{R}_{+} \rightarrow \mathbf{R}_{+}$is a strictly increasing differentiable function with $\varphi(0)=0$ and $\lim _{x \rightarrow \infty} \varphi(x)=\infty$, then the operator

$$
\left(U_{\varphi} f\right)(x)=f(\varphi(x)) \sqrt{\varphi^{\prime}(x)}
$$

is a unitary operator from $L^{2}\left(\mathbf{R}_{+}\right)$to itself which furthermore satisfies the identity $U_{\varphi}^{-1}=U_{\varphi^{-1}}$. Under conjugation with $U_{\varphi}$, we see that one has the unitary equivalences

$$
\partial_{x} \approx \frac{1}{\varphi^{\prime}(x)} \partial_{x}-\frac{\varphi^{\prime \prime}(x)}{2\left(\varphi^{\prime}(x)\right)^{2}}, \quad V(x) \approx V(\varphi(x)) .
$$

Choosing $\varphi(x)=x^{2 /(2-\gamma)}$, we see that $\mathcal{L}_{1}$ is unitarily equivalent to the operator $\mathcal{L}_{2}$ given by

$$
\begin{aligned}
\left(\mathcal{L}_{2} f\right)(x) & =\frac{1}{2}\left(\frac{2-\gamma}{2} \partial_{x}+\frac{\gamma}{4 x}\right)\left(\frac{2-\gamma}{2} \partial_{x}-\frac{\gamma}{4 x}\right)-\frac{x^{\frac{2 \gamma-2}{2-\gamma}}}{2}\left(x^{\frac{2}{2-\gamma}}-\gamma\right) \\
& =\frac{(2-\gamma)^{2}}{8} \partial_{x}^{2}+\left(\frac{\gamma(2-\gamma)}{16}-\frac{\gamma^{2}}{32}\right) x^{-2}-\frac{x^{\frac{2 \gamma-2}{2-\gamma}}}{2}\left(x^{\frac{2}{2-\gamma}}-\gamma\right)
\end{aligned}
$$

This is a Schrödinger operator with a potential that behaves at infinity like $x^{2 \gamma /(2-\gamma)}$. It follows that

- If $1>\gamma>0$, then the operator $\mathcal{L}_{2}$ has compact resolvent.

- If $\gamma=0$, the operator $\mathcal{L}_{2}$ does not have compact resolvent, but it still has a spectral gap (since one can see that its essential spectrum is the interval $[1 / 2, \infty)$ ).

- If $\gamma<0$, then 0 belongs to the essential spectrum of $\mathcal{L}_{2}$.

See for example [RS78] for a proof. It is then a natural conjecture that the spectrum of the generator of (1.5) on the $L^{2}$ space weighted by the invariant measure has the same behavior (as a function of the parameter $\gamma=\frac{2 n}{k}+1-2 n$ ) as just described. The next section is a step towards a proof of this conjecture.

\section{Lack of spectral gap}

The aim of this section is to obtain information on the location of the essential spectrum of the generator $\mathcal{L}$ for (1.5). This will be accomplished by using ideas from averaging/homogenization theory to build a set of approximate eigenvectors. Since $\mathcal{L}$ is not self-adjoint, there are various possible definitions of its essential spectrum (see [EE87] or [GW69] for a survey). We choose to retain the following definition:

Definition 3.1 For $T$ a closed densely defined operator on a Banach space $\mathcal{B}$, the essential spectrum $\sigma_{e}(T)$ is defined as the set of all values $\lambda \in \mathbf{C}$ such that $T-\lambda$ is not a semi-Fredholm operator. 
The set $\sigma_{e}(T)$ is contained in the corresponding sets for all other alternative definitions of the essential spectrum appearing in the abovementioned works. In this sense, the statement " $\lambda \in \sigma_{e}(T)$ " used here is the strongest. In particular, it is contained in the set

$$
\bigcap_{K \in \mathcal{K}(\mathcal{B})} \sigma(T+K),
$$

where $\mathcal{K}(\mathcal{B})$ denotes the ideal of all compact operators on $\mathcal{B}$ and $\sigma(T)$ denotes the spectrum of an operator $T$.

We will make use of the following generalization of Weyl's criterion [RS78], which gives a useful criterion for identifying the essential spectrum:

Proposition 3.2 Let $T$ be a closed densely defined operator on a Banach space B. For any $\lambda \in \mathbf{C}, \lambda \in \sigma_{e}(T)$ if and only if there exist sequences $\varphi_{n}$ and $\varphi_{n}^{*}$ of elements in $\mathcal{D}(T) \subset \mathcal{B}$ and $\mathcal{D}\left(T^{*}\right) \subset \mathcal{B}^{*}$ respectively with $\left\|\varphi_{n}\right\|=\left\|\varphi_{n}^{*}\right\|=1$ and having no convergent subsequence such that $\lim _{n \rightarrow \infty}\left\|T \varphi_{n}-\lambda \varphi_{n}\right\|=\lim _{n \rightarrow \infty} \| T^{*} \varphi_{n}^{*}-$ $\bar{\lambda} \varphi_{n}^{*} \|=0$.

Proof. See [EE87, Theorem 9.1.3] and [Kat95, Theorem IV.5.11], or the original work [Wol59].

There are situations in which, even though it is difficult to locate the essential spectrum precisely, one can nevertheless exhibit a sequence $\varphi_{n}$ as above such that $T \varphi_{n}$ remains bounded. In that case, one has:

Proposition 3.3 Let $T$ be a closed densely defined operator on a Banach space $\mathcal{B}$ with non-empty resolvent set. If there exists a sequence $\varphi_{n}$ of elements in $\mathcal{D}(T) \subset \mathcal{B}$ with $\left\|\varphi_{n}\right\|=1$ and having no convergent subsequence such that $\lim \sup _{n \rightarrow \infty}\left\|T \varphi_{n}\right\|<\infty$, then $T$ does not have compact resolvent.

Proof. This claim follows from the fact that the compactness of the resolvent is equivalent to the statement that sets of the form $\{\varphi \mid\|\varphi\| \leq 1 \&\|T \varphi\| \leq K\}$ are precompact.

\subsection{Scalings}

Before we turn to the study of the generator, we make some remarks about the regularity and the behavior of functions that scale in a particular way.

Denote by $X_{H_{f}}=P \partial_{Q}-Q|Q|^{2 k-2} \partial_{P}$ the Liouville operator associated to the "free" oscillator

$$
H_{f}(P, Q)=\frac{P^{2}}{2}+\frac{|Q|^{2 k}}{2 k} .
$$

The constant $k$ is not necessarily an integer, so that in general the function $H_{f}$ is not $\mathcal{C}^{\infty}$ but only $\mathcal{C}^{[2 k]}$, where $[2 k]$ is the integer part of $2 k$. We introduce the following definition:

Definition 3.4 A function $\psi: \mathbf{R}^{2} \backslash\{0\} \rightarrow \mathbf{R}$ is said to scale like $H_{f}^{\alpha}$ if it satisfies the relation $\psi\left(\lambda P, \lambda^{\frac{1}{k}} Q\right)=\lambda^{2 \alpha} \psi(P, Q)$.

One has the following elementary result: 
Lemma 3.5 If $\psi \in \mathcal{C}^{1}\left(\mathbf{R}^{2} \backslash\{0\}\right)$ scales like $H_{f}^{\alpha}$, then $\partial_{P} \psi$ scales like $H_{f}^{\alpha-\frac{1}{2}}$, $\partial_{Q} \psi$ scales like $H_{f}^{\alpha-\frac{1}{2 k}}$, and $X_{H_{f}} \psi$ scales like $H_{f}^{\alpha+\frac{1}{2}-\frac{1}{2 k}}$.

Given some fixed $\alpha$, there is a one-to-one correspondence between functions $\varphi$ that scale like $H_{f}{ }^{\alpha}$ and functions on the circle $S^{1}$ in the following way. Define a function $r: S^{1} \rightarrow \mathbf{R}_{+}$by the unique positive solution to

$$
\frac{r^{2}(\theta) \cos ^{2} \theta}{2}+\frac{r^{2 k}(\theta)|\sin \theta|^{2 k}}{2 k}=1,
$$

and set

$$
(\mathcal{S} \varphi)(\theta)=\varphi(r(\theta) \cos \theta, r(\theta) \sin \theta) .
$$

The function $r$ is bounded away from 0 (it is actually between $\sqrt{2}$ and $(2 k)^{1 /(2 k)}$ and, by the implicit functions theorem, it is of class $\mathcal{C}^{[2 k]}$. A straightforward but slightly tedious calculation shows that, via $\mathcal{S}$, the operator $X_{H_{f}}$ is conjugated to the differential operator

$$
\left(\tilde{X}_{H_{f}} f\right)(\theta)=\omega(\theta) f^{\prime}(\theta), \quad \omega(\theta)=\cos ^{2} \theta+r^{2 k-2}(\theta)|\sin \theta|^{2 k} .
$$

Definition 3.6 A function $\psi: \mathbf{R}^{2} \rightarrow \mathbf{R}$ that scales like $H_{f}{ }^{\alpha}$ is said to average out to 0 if

$$
\int_{0}^{2 \pi} \frac{(\mathcal{S} \psi)(\theta)}{\omega(\theta)} d \theta=0
$$

With these preliminaries, the following result is now straightforward:

Proposition 3.7 Let $\psi \in \mathcal{C}^{r}\left(\mathbf{R}^{2} \backslash\{0\}\right)$ scale like $H_{f}^{\alpha}$ and average out to 0 . Then, there exists a unique solution $\varphi$ to the equation

$$
X_{H_{f}} \varphi=\psi,
$$

such that $\varphi$ also averages out to 0 . Furthermore, $\varphi$ scales like $H_{f}^{\alpha+\frac{1}{2 k}-\frac{1}{2}}$ and one has $\varphi \in \mathcal{C}^{r^{\prime}}\left(\mathbf{R}^{2} \backslash\{0\}\right)$ with $r^{\prime}=\min \{[2 k], r+1\}$.

Proof. Let $\varphi$ be the unique function scaling like $H_{f}{ }^{\alpha+\frac{1}{2 k}-\frac{1}{2}}$ and such that

$$
(\mathcal{S} \varphi)(\theta)=\int_{0}^{\theta} \frac{(\mathcal{S} \psi)(t)}{\omega(t)} d t-\frac{1}{2 \pi} \int_{0}^{2 \pi} \int_{0}^{\theta} \frac{(\mathcal{S} \psi)(t)}{\omega(t)} d t d \theta .
$$

One can check that one has indeed $X_{H_{f}} \varphi=\psi$. Furthermore, it follows from their explicit expressions that both $\mathcal{S}$ and $\mathcal{S}^{-1}$ map $\mathcal{C}^{r}$ functions into $\mathcal{C}^{r}$ functions as long as $r \leq[2 k]$.

We conclude this section with a small lemma that allows us to compute the $L^{2}$ norm of functions that scale in a certain way. Let $(P, Q) \in \mathbf{R}^{2}$ and $(x, y) \in \mathbf{R}^{2 n}$ for some $n \geq 1$. The functions that will be considered in the remainder of this section will always be of the form

$$
\varphi_{\mathcal{E}}(P, Q, x, y)=F(x-g(P, Q), y-h(P, Q)) \psi(P, Q) \chi\left(H_{f}(P, Q) / \mathcal{E}\right),
$$

for some parameter $\mathcal{E}>0$. One has 
Lemma 3.8 Let $F \in L^{2}\left(\mathbf{R}^{2 n}\right)$, let $g, h: \mathbf{R}^{2} \rightarrow \mathbf{R}^{n}$ be measurable, let $\chi: \mathbf{R}_{+} \rightarrow \mathbf{R}$ be continuous and compactly supported away from 0 , and let $\psi$ be a function that is continuous away from 0 and scales like $H_{f}^{\alpha}$ for some $\alpha \in \mathbf{R}$. Then, the $L^{2}$-norm of $\varphi_{\mathcal{E}}$ defined as in (3.3) satisfies $\left\|\varphi_{\mathcal{E}}\right\| \propto \mathcal{E}^{\alpha+\frac{1}{4}+\frac{1}{4 k}}$.

Proof. Make the change of variables $(\tilde{P}, \tilde{Q})=\left(\mathcal{E}^{\frac{1}{2}} P, \mathcal{E}^{\frac{1}{2 k}} Q\right)$ and use the scaling properties of $\psi$.

\subsection{The case of three oscillators}

Before we tackling the general case of a chain with arbitrary length, let us "cut our teeth" on the problem with three oscillators. Since we do not have an explicit expression for the invariant measure $\mu$ (indeed, at this stage, we do not even know that it exists!), we are going to study the generator of (1.5) in spaces of the type $L^{2}\left(e^{-\beta H} d p d q\right)$. As in [EPR99b, EH00], it is not expected that the qualitative nature of the spectrum of $\mathcal{L}$ depends on the choice of $\beta$, as long as $\beta<2 \min \left\{\beta_{0}, \beta_{2}\right\}$ (as usual, we set $\beta_{i}=1 / T_{i}$ ). Since one expects the true invariant measure to be somehow "in between" the Gibbs measures at temperatures $T_{0}$ and $T_{2}$, it is very likely that the qualitative nature of the spectrum of $\mathcal{L}$ in $L^{2}(\mu)$ is also the same.

We write

$$
\begin{aligned}
& H_{0}=\frac{p_{0}^{2}}{2}+\frac{\left|q_{0}\right|^{2 k}}{2 k}+\frac{q_{0}^{2}}{2}, \quad H_{2}=\frac{p_{2}^{2}}{2}+\frac{\left|q_{2}\right|^{2 k}}{2 k}+\frac{q_{2}^{2}}{2}, \\
& H_{1}=\frac{p_{1}^{2}}{2}+\frac{\left|q_{1}\right|^{2 k}}{2 k} .
\end{aligned}
$$

Remark 3.9 One should not think of $H_{0}$ (and $H_{2}$ ) as the energy one gets by pinning $q_{1}$ at 0 , but rather as the energy such that the corresponding force is the one that gets averaged out over the fast motion of the middle oscillator.

With this notation, the Liouville operator $X_{H}=\partial_{p} H \partial_{q}-\partial_{q} H \partial_{p}$ for the total Hamiltonian can be broken up as follows:

$$
X_{H}=X_{H_{0}}+X_{H_{2}}+X_{H_{1}}+\left(q_{0}+q_{2}-2 q_{1}\right) \partial_{p_{1}}+q_{1}\left(\partial_{p_{0}}+\partial_{p_{2}}\right) .
$$

Recall that the generator of the stochastic dynamics is given by

$$
\mathcal{L}=X_{H}-\gamma_{0} p_{0} \partial_{p_{0}}+\gamma_{0} T_{0} \partial_{p_{0}}^{2}-\gamma_{2} p_{2} \partial_{p_{2}}+\gamma_{2} T_{2} \partial_{p_{2}}^{2} .
$$

The space $L^{2}\left(e^{-\beta H} d p d q\right)$ isometric to $L^{2}$ via the operator $K f=e^{-\beta H / 2} f$. This shows that $\mathcal{L}$ is conjugate to the operator $\tilde{\mathcal{L}}=K \mathcal{L} K^{-1}$ on the flat $L^{2}$ space given by

$$
\begin{aligned}
\tilde{\mathcal{L}} & =X_{H}+\sum_{i=0,2} \gamma_{i} T_{i}\left(\left(\alpha_{i}-\alpha_{i}^{*}\right) p_{i} \partial_{p_{i}}+\partial_{p_{i}}^{2}-\alpha_{i} \alpha_{i}^{*} p_{i}^{2}+\alpha_{i}\right) \\
& =X_{H}+\gamma_{0} T_{0} \mathcal{L}_{\mathrm{ou}}^{0}+\gamma_{2} T_{2} \mathcal{L}_{\mathrm{oU}}^{2} .
\end{aligned}
$$

where we set

$$
\alpha_{i}=\frac{\beta}{2}, \quad \alpha_{i}^{*}=\frac{1}{T_{i}}-\frac{\beta}{2} .
$$

Remark 3.10 Here we see the importance of the condition $\beta<2 \min \left\{\beta_{0}, \beta_{2}\right\}$ : it makes sure that the coefficients in front of $p_{i}^{2}$ are strictly negative. If this is not the case, $\tilde{\mathcal{L}}$ is not dissipative anymore and does therefore not generate a $C_{0}$-semigroup on $L^{2}\left(e^{-\beta H} d p d q\right)$. 
The main result of this section is:

Theorem 3.11 If $k \geq 2$, then the operator $\tilde{\mathcal{L}}$ does not have compact resolvent for any $\beta<2 \min \left\{\beta_{0}, \beta_{2}\right\}$. If $k>2$, then it has essential spectrum at 0 .

Proof. The aim is to construct a sequence $\varphi_{n}$ of approximate eigenfunctions such that all the $\varphi_{n}$ are mutually orthogonal, $\left\|\varphi_{n}\right\|=1$ and $\left\|\tilde{\mathcal{L}} \varphi_{n}\right\|$ either stays bounded or converges to 0 . By Propositions 3.2 and 3.3, this would then immediately imply a lack of compactness for the resolvent of $\tilde{\mathcal{L}}$, or even the presence of essential spectrum at 0 . Since the spectral properties of $\tilde{\mathcal{L}}$ and of its adjoint $\tilde{\mathcal{L}}^{*}$ are the same, we can also construct such a sequence of approximate eigenfunctions for $\tilde{\mathcal{L}}^{*}$ instead. They can then be interpreted as approximate invariant measures for the dynamic (1.5). Since it seems to be a little bit easier to get an intuition about densities of approximate invariant measures rather than about approximately invariant observables, this is what we are going to do in this section.

The adjoint of $\tilde{\mathcal{L}}$ is given by

$$
\begin{aligned}
\tilde{\mathcal{L}}^{*} & =-X_{H}+\gamma_{0} T_{0}\left(\mathcal{L}_{\mathrm{oU}}^{0}\right)^{*}+\gamma_{2} T_{2}\left(\mathcal{L}_{\mathrm{oU}}^{2}\right)^{*} \\
\left(\mathcal{L}_{\mathrm{oU}}^{i}\right)^{*} & =\left(\alpha_{i}^{*}-\alpha_{i}\right) p_{i} \partial_{p_{i}}+\partial_{p_{i}}^{2}-\alpha_{i} \alpha_{i}^{*} p_{i}^{2}+\alpha_{i}^{*} .
\end{aligned}
$$

Direct calculation shows that $\exp \left(-\alpha_{i} H_{i}\right)$ is an eigenfunction with eigenvalue 0 for $\mathcal{L}_{\text {OU }}^{i}$ and $\exp \left(-\alpha_{i}^{*} H_{i}\right)$ is an eigenfunction with eigenvalue 0 for $\left(\mathcal{L}_{\text {oU }}^{i}\right)^{*}$ with $i \in\{0,2\}$. However, if $T_{0} \neq T_{2}$ (and therefore $\alpha_{0} \neq \alpha_{2}$ ) it is not possible in general to find a closed expression for an eigenfunction with eigenvalue 0 for $\tilde{\mathcal{L}}^{*}$. Note also that $\alpha_{i}=\alpha_{i}^{*}$ if and only if $T_{i}=1 / \beta$, which is not surprising since in this case $\mathcal{L}_{\mathrm{OU}}^{i}$ is self-adjoint.

Choose now a function $\chi: \mathbf{R} \rightarrow[0,1]$ which is smooth and compactly supported on $[1,2]$ and set for example $\mathcal{E}_{n}=3^{n}$, so that the functions $\chi\left(H_{1} / \mathcal{E}_{n}\right)$ have disjoint support. The formal calculation performed in Section 2 suggests that when the energy of the middle oscillator is large, the dynamic of (1.5) keeps that energy approximately constant, while the two boundary oscillators equilibrate approximately at Gibbs measures at temperatures $T_{0}$ and $T_{2}$ respectively. Our first guess would be therefore to build approximate eigenfunctions for $\tilde{\mathcal{L}}$ and $\tilde{\mathcal{L}}^{*}$ by setting

$$
\varphi_{n}=C_{n} e^{-\alpha_{0} H_{0}-\alpha_{2} H_{2}} \chi\left(H_{1} / \mathcal{E}_{n}\right), \quad \varphi_{n}^{*}=C_{n}^{*} e^{-\alpha_{0}^{*} H_{0}-\alpha_{2}^{*} H_{2}} \chi\left(H_{1} / \mathcal{E}_{n}\right) .
$$

Here, the constants $C_{n}$ and $C_{n}^{*}$ are chosen such that $\left\|\varphi_{n}\right\|=\left\|\varphi_{n}^{*}\right\|=1$. From Lemma 3.8, one infers that $C_{n} \propto C_{n}^{*} \propto \mathcal{E}_{n}^{-\frac{1}{4 k}-\frac{1}{4}}$. With this guess, we get

$$
\tilde{\mathcal{L}} \varphi_{n}=C_{n} e^{-\alpha_{0} H_{0}-\alpha_{2} H_{2}}\left(\frac{p_{1}\left(q_{0}+q_{2}-2 q_{1}\right)}{\mathcal{E}_{n}} \chi^{\prime}\left(\frac{H_{1}}{\mathcal{E}_{n}}\right)-q_{1}\left(\alpha_{0} p_{0}+\alpha_{2} p_{2}\right) \chi\left(\frac{H_{1}}{\mathcal{E}_{n}}\right)\right),
$$

and similarly for $\tilde{\mathcal{L}}^{*} \varphi_{n}^{*}$. Since $p_{1} \approx \mathcal{E}_{n}^{\frac{1}{2}} \ll \mathcal{E}_{n}$ (on the support of $\varphi_{n}$ ), the first term goes to 0 in $L^{2}$. The second term however goes to $\infty$ because of the factor $q_{1}$, so we have to be a little bit more careful in our analysis.

The problem is that we have not so far exploited the fact that we also know approximately what the fast oscillations superimposed over the slow dynamic of the boundary oscillators look like, see (2.5). These oscillations can be expressed as a function $\Phi$ of the middle oscillator, solution to the Poisson equation

$$
X_{H_{f}} \Phi(P, Q)=-Q .
$$


By Proposition 3.7, this equation has a unique solution that averages to 0 along orbits of the solutions corresponding to $H_{f}$. Furthermore, $\Phi$ scales like $H_{f}^{\frac{1}{k}-\frac{1}{2}}$. In particular, we note that $\Phi$ is bounded when $k=2$ and converges to 0 at large energies when $k>2$.

Our next guess is therefore to compensate for these fast oscillations by setting $\bar{p}_{i}=$ $p_{i}+\Phi\left(p_{1}, q_{1}\right)$ and taking

$$
\varphi_{n}=C_{n} \exp \left(-\alpha_{0} H_{0}\left(\bar{p}_{0}, q_{0}\right)-\alpha_{2} H_{2}\left(\bar{p}_{2}, q_{2}\right)\right) \chi\left(\frac{H_{1}}{\mathcal{E}_{n}}\right) .
$$

Observe at this stage that $H_{1}=H_{f}\left(p_{1}, q_{1}\right)$, so that we can make use of (3.4) when computing $X_{H_{1}} \varphi_{n}$. One has, for $i=0,2$ :

$$
\begin{aligned}
X_{H_{i}} \varphi_{n} & =-\alpha_{i}\left(q_{i}\left|q_{i}\right|^{2 k-2}+q_{i}\right) \Phi \varphi_{n} \\
X_{H_{1}} \varphi_{n} & =\alpha_{0} \bar{p}_{0} q_{1} \varphi_{n}+\alpha_{2} \bar{p}_{2} q_{1} \varphi_{n} \\
q_{1}\left(\partial_{p_{0}}+\partial_{p_{2}}\right) \varphi_{n} & =-\alpha_{0} \bar{p}_{0} q_{1} \varphi_{n}-\alpha_{2} \bar{p}_{2} q_{1} \varphi_{n} \\
\left(q_{0}+q_{2}-2 q_{1}\right) \partial_{p_{1}} \varphi_{n} & =\left(q_{0}+q_{2}-2 q_{1}\right)\left(-\left(\alpha_{0} \bar{p}_{0}+\alpha_{2} \bar{p}_{2}\right) \partial_{P} \Phi+\frac{p_{1}}{\mathcal{E}_{n}} \frac{\chi^{\prime}}{\chi}\right) \varphi_{n}, \\
\mathcal{L}_{\mathrm{oU}}^{i} \varphi_{n} & =\alpha_{i} \Phi\left(\left(\alpha_{i}+\alpha_{i}^{*}\right) p_{i}+\alpha_{i} \Phi\right) \varphi_{n} .
\end{aligned}
$$

Here, we have omitted the argument $\left(p_{1}, q_{1}\right)$ of $\Phi$ and the argument $H_{1} / \mathcal{E}$ of $\chi$ and $\chi^{\prime}$ for the sake of simplicity. Note now that (3.5) and (3.6) cancel each other out exactly. It follows from Lemma 3.8 that

$$
\begin{aligned}
\left\|X_{H_{i}} \varphi_{n}\right\| & \lesssim \mathcal{E}_{n}^{\frac{1}{k}-\frac{1}{2}}, \\
\left\|\left(q_{0}+q_{2}-2 q_{1}\right) \partial_{p_{1}} \varphi_{n}\right\| & \lesssim\left(1+\mathcal{E}_{n}^{\frac{1}{2 k}}\right)\left(\mathcal{E}_{n}^{\frac{1}{k}-1}+\mathcal{E}_{n}^{-\frac{1}{2}}\right) \lesssim \mathcal{E}_{n}^{\frac{1}{2 k}-\frac{1}{2}}, \\
\left\|\mathcal{L}_{\mathrm{OU}}^{i} \varphi_{n}\right\| & \lesssim \mathcal{E}_{n}^{\frac{1}{k}-\frac{1}{2}}+\mathcal{E}_{n}^{\frac{2}{k}-1} \lesssim \mathcal{E}_{n}^{\frac{1}{k}-\frac{1}{2}} .
\end{aligned}
$$

Note that the exponent $\frac{1}{4}+\frac{1}{4 k}$ appearing in Lemma 3.8 is precisely canceled by the normalization constant $C_{n}$. We also used here the symbol $\Psi_{1} \lesssim \Psi_{2}$ for two expressions $\Psi_{i}$ as a shorthand for "there exists a constant $C$ such that $\Psi_{1} \leq C \Psi_{2}$." It follows from the above bounds that $\left\|\mathcal{L} \varphi_{n}\right\| \lesssim \mathcal{E}_{n}^{\frac{1}{k}-\frac{1}{2}}$.

It is possible to construct approximate eigenfunctions $\varphi_{n}^{*}$ for $\mathcal{L}^{*}$ similarly by setting

$$
\varphi_{n}^{*}=C_{n} \exp \left(-\alpha_{0}^{*} H_{0}\left(\bar{p}_{0}, q_{0}\right)-\alpha_{2}^{*} H_{2}\left(\bar{p}_{2}, q_{2}\right)\right) \chi\left(\frac{H_{1}}{\mathcal{E}_{n}}\right) \text {. }
$$

Note now that the only difference between $\mathcal{L}$ and $\mathcal{L}^{*}$ is that one changes the sign of $X_{H}$ and switches $\alpha_{i}$ and $\alpha_{i}^{*}$. This shows that the cancellation between (3.5) and (3.6) still takes place when applying $\mathcal{L}^{*}$ to $\varphi_{n}^{*}$, so that $\left\|\mathcal{L}^{*} \varphi_{n}^{*}\right\| \lesssim \mathcal{E}_{n}^{\frac{1}{k}-\frac{1}{2}}$ as above.

If $k=2$, it follows that there exists a constant $C$ such that $\left\|\mathcal{L} \varphi_{n}\right\|+\left\|\mathcal{L}^{*} \varphi_{n}^{*}\right\| \leq C$ for every $n$. If $k>2$, all the exponents appearing the the above expressions are negative, so that $\lim _{n \rightarrow \infty}\left(\left\|\mathcal{L} \varphi_{n}\right\|+\left\|\mathcal{L}^{*} \varphi_{n}^{*}\right\|\right)=0$. Applying Propositions 3.2 and 3.3 concludes the proof of the theorem.

Remark 3.12 It is clear from the proof that the exact same result also holds for a chain consisting of 4 oscillators instead of 3 . One can construct approximate invariant measures in exactly the same way, but one has the additional freedom of choosing to take the energy of either of the two middle oscillators to be large. 


\subsection{Longer chain}

In this section, we consider a chain of length $N+1$ for $N \geq 4$. We will show that if $k>\frac{3}{2}$, then the generator of the dynamic has essential spectrum at 0 . To this end, define similarly as before

$$
\begin{aligned}
& H_{0}=\frac{p_{0}^{2}+p_{1}^{2}}{2}+\frac{\left|q_{0}\right|^{2 k}+\left|q_{1}\right|^{2 k}}{2 k}+\frac{\left(q_{0}-q_{1}\right)^{2}+q_{1}^{2}}{2}, \\
& H_{r}=\frac{q_{3}^{2}}{2}+\sum_{i=3}^{N}\left(\frac{p_{i}^{2}}{2}+\frac{\left|q_{i}\right|^{2 k}}{2 k}\right)+\sum_{i=4}^{N} \frac{\left(q_{i}-q_{i-1}\right)^{2}}{2}, \\
& H_{c}=\frac{p_{2}^{2}}{2}+\frac{\left|q_{2}\right|^{2 k}}{2},
\end{aligned}
$$

so that

$$
X_{H}=X_{H_{0}}+X_{H_{r}}+X_{H_{c}}+\left(q_{1}+q_{3}-2 q_{2}\right) \partial_{p_{2}}+q_{2}\left(\partial_{p_{1}}+\partial_{p_{3}}\right) .
$$

As in the previous section, we consider the operator $\tilde{\mathcal{L}}$ on the flat $L^{2}$ space given by

$$
\tilde{\mathcal{L}}=X_{H}+\gamma_{0} T_{0} \mathcal{L}_{\text {ou }}^{0}+\gamma_{N} T_{N} \mathcal{L}_{\text {ou }}^{r} .
$$

We have

Theorem 3.13 If $N+1 \geq 5$ and $k>\frac{3}{2}$, then the operator $\tilde{\mathcal{L}}$ has essential spectrum at 0 . If $k=\frac{3}{2}$, it does not have compact resolvent. As previously, these statements are independent of the value of $\beta<2 \min \left\{\beta_{0}, \beta_{N}\right\}$.

Proof. Let $\Phi$ be defined as in the previous subsection. In this section, we do not only add a corrector term to $p_{1}$ and $p_{3}$, but also to $q_{1}$ and $q_{3}$. We define $\Phi^{(2)}$ to be the solution to the Poisson equation $X_{H_{f}} \Phi^{(2)}=\Phi$ and we define new variables $\bar{p}$ and $\bar{q}$ by

$$
\bar{p}_{i}=p_{i}+\Phi\left(p_{2}, q_{2}\right), \quad \bar{q}_{i}=q_{i}+\Phi^{(2)}\left(p_{2}, q_{2}\right),
$$

for $i=1,3$ and $\left(\bar{p}_{i}, \bar{q}_{i}\right)=\left(p_{i}, q_{i}\right)$ otherwise. With this notation, we set as before

$$
\varphi_{n}=C_{n} \exp \left(-\alpha_{0} H_{0}(\bar{p}, \bar{q})-\alpha_{N} H_{r}(\bar{p}, \bar{q})\right) \chi\left(H_{c} / \mathcal{E}_{n}\right) .
$$

In order to compute $X_{H} \varphi_{n}$, let us first apply $X_{H}$ to $\bar{p}_{i}$ and $\bar{q}_{i}$ :

$$
\begin{aligned}
X_{H} \bar{q}_{0}= & \bar{p}_{0} \\
X_{H} \bar{p}_{0}= & -\bar{q}_{0}\left|\bar{q}_{0}\right|^{2 k-2}+\bar{q}_{1}-\bar{q}_{0}-\Phi^{(2)} \\
X_{H} \bar{q}_{1}= & \bar{p}_{1}+\left(q_{0}+q_{2}-2 q_{1}\right) \partial_{P} \Phi^{(2)} \\
X_{H} \bar{p}_{1}= & -\bar{q}_{1}\left|\bar{q}_{1}\right|^{2 k-2}+\bar{q}_{0}-2 \bar{q}_{1} \\
& +\left(\bar{q}_{1}\left|\bar{q}_{1}\right|^{2 k-2}-q_{1}\left|q_{1}\right|^{2 k-2}\right)+\left(q_{0}+q_{2}-2 q_{1}\right) \partial_{P} \Phi+2 \Phi^{(2)} .
\end{aligned}
$$

Hence

$$
\begin{aligned}
X_{H} H_{0}(\bar{p}, \bar{q})= & -\bar{p}_{0} \Phi^{(2)}-\left(\bar{q}_{1}\left|\bar{q}_{1}\right|^{2 k-1}+2 \bar{q}_{1}-\bar{q}_{0}\right)\left(q_{0}+q_{2}-2 q_{1}\right) \partial_{P} \Phi^{(2)} \\
& +\bar{p}_{1}\left(\bar{q}_{1}\left|\bar{q}_{1}\right|^{2 k-2}-q_{1}\left|q_{1}\right|^{2 k-2}\right)+\bar{p}_{1}\left(q_{0}+q_{2}-2 q_{1}\right) \partial_{P} \Phi+2 \bar{p}_{1} \Phi^{(2)} \\
= & R_{1}+R_{2}+R_{3}+R_{4}+R_{5}
\end{aligned}
$$


and similarly for $X_{H} H_{r}(\bar{p}, \bar{q})$ by symmetry. We have furthermore

$$
X_{H} H_{c}=p_{2}\left(q_{1}+q_{3}-2 q_{2}\right),
$$

so that

$$
X_{H} \chi\left(H_{c} / \mathcal{E}_{n}\right)=\frac{q_{1}+q_{3}-2 q_{2}}{\mathcal{E}_{n}} p_{2} \chi^{\prime}\left(H_{c} / \mathcal{E}_{n}\right) .
$$

Since $\mathcal{L}_{\text {ou }}^{0} \varphi_{n}=\mathcal{L}_{\text {ou }}^{r} \varphi_{n}=0$, we thus have

$$
\tilde{\mathcal{L}} \varphi_{n}=\left(-\alpha_{0} X_{H} H_{0}(\bar{p}, \bar{q})-\alpha_{N} X_{H} H_{r}(\bar{p}, \bar{q})+\frac{X_{H} \chi\left(H_{c} / \mathcal{E}_{n}\right)}{\chi\left(H_{c} / \mathcal{E}_{n}\right)}\right) \varphi_{n} .
$$

We bound the terms appearing in this expression in the same way as in the previous subsection. Since $\Phi$ scales like $H_{f}^{\frac{1}{k}-\frac{1}{2}}$ and $\Phi^{(2)}$ scales like $H_{f}^{\frac{3}{2 k}-1}$, Lemma 3.8 shows that

$$
\begin{aligned}
\left\|R_{1} \varphi_{n}\right\| & \lesssim \mathcal{E}_{n}^{\frac{3}{2 k}-1}, \quad\left\|R_{2} \varphi_{n}\right\| & \lesssim\left(1+\mathcal{E}_{n}^{\frac{1}{2 k}}\right) \mathcal{E}_{n}^{\frac{3}{2 k}-\frac{3}{2}} \lesssim \mathcal{E}_{n}^{\frac{2}{k}-\frac{3}{2}}, \\
\left\|R_{3} \varphi_{n}\right\| & \lesssim \mathcal{E}_{n}^{\frac{3}{2 k}-1}, \quad\left\|R_{4} \varphi_{n}\right\| \lesssim\left(1+\mathcal{E}_{n}^{\frac{1}{2 k}}\right) \mathcal{E}_{n}^{\frac{1}{h}}-1 & \lesssim \mathcal{E}_{n}^{\frac{3}{2 k}-1}, \\
\left\|R_{5} \varphi_{n}\right\| & \lesssim \mathcal{E}_{n}^{\frac{3}{2 k}-1}, \quad\left\|\frac{X_{H} \chi}{\chi} \varphi_{n}\right\| & \lesssim\left(1+\mathcal{E}_{n}^{\frac{1}{2 k}}\right) \mathcal{E}_{n}^{-\frac{1}{2}} \lesssim \mathcal{E}_{n}^{\frac{1}{2 k}-\frac{1}{2}}
\end{aligned}
$$

Collecting all these bounds, we obtain $\left\|\tilde{\mathcal{L}} \varphi_{n}\right\| \lesssim \max \left\{\mathcal{E}_{n}^{\frac{3}{2 k}-1}, \mathcal{E}_{n}^{\frac{1}{2 k}-\frac{1}{2}}\right\}$. As before, if we set

$$
\varphi_{n}^{*}=C_{n} \exp \left(-\alpha_{0}^{*} H_{0}(\bar{p}, \bar{q})-\alpha_{N}^{*} H_{r}(\bar{p}, \bar{q})\right) \chi\left(H_{c} / \mathcal{E}_{n}\right),
$$

we obtain the same bounds for $\left\|\tilde{\mathcal{L}}^{*} \varphi_{n}^{*}\right\|$. The exponents appearing in all of these bounds are strictly negative whenever $k>3 / 2$, thus concluding the proof of Theorem 3.13.

\section{Effective dynamics}

From now on, we study the case of three oscillators in detail. In this section, we derive an effective dynamic for the outer oscillators that is valid in the regime where most of the energy is located in the center oscillator. More precisely, we show that there exists a change of variable $\left(p_{i}, q_{i}\right) \mapsto\left(\bar{p}_{i}, \bar{q}_{i}\right)$ for $i=0,2$ such that the equations of motion for $\left(\bar{p}_{i}, \bar{q}_{i}\right)$ decouple (to leading order) from the rest of the system, provided that the energy of the middle oscillator is large.

As before, we will use throughout this section the symbol $\Psi_{1} \lesssim \Psi_{2}$ for two expressions $\Psi_{i}$ as a shorthand for "there exists a constant $C$ such that $\Psi_{1} \leq C \Psi_{2}$." The constant $C$ depends in general on the parameters of the model, but is of course independent of the arguments of the $\Psi_{i}$.

Theorem 4.1 Assume that $k>\frac{3}{2}$. There exist smooth functions $\Phi_{p}^{i}$ and $\Phi_{q}^{i}$ depending on $\left(p_{i}, q_{i}, p_{1}, q_{1}\right)$ such that if we make the change of variables $\bar{p}_{i}=p_{i}+\Phi_{p}^{i}$ and $\bar{q}_{i}=q_{i}+\Phi_{q}^{i}($ for $i=0,2)$, the equations of motion for $\left(\bar{p}_{i}, \bar{q}_{i}\right)$ are given by

$$
\begin{aligned}
d \bar{q}_{i} & =\bar{p}_{i} d t+R_{q}^{i} d t+\Sigma_{q}^{i} d w_{i} \\
d \bar{p}_{i} & =-\bar{q}_{i}\left|\bar{q}_{i}\right|^{2 k-2} d t-\bar{q}_{i} d t-\gamma_{i} \bar{p}_{i} d t+R_{p}^{i} d t+\Sigma_{p}^{i} d w_{i}
\end{aligned}
$$


for some adapted processes $R_{p}^{i}, R_{q}^{i}, \Sigma_{p}^{i}$ and $\Sigma_{q}^{i}$. Furthermore, the error terms $R$ and $\Sigma$ satisfy the bounds

$$
\begin{array}{ll}
\left|R_{p}^{i}\right| \lesssim\left(\bar{E}_{0}+\bar{E}_{2}\right)^{\frac{1}{2}-\delta} & \left|R_{q}^{i}\right| \lesssim \bar{E}_{i}^{\frac{1}{2 k}-\delta}+\frac{\bar{E}_{2-i}^{\frac{1}{2 k}}}{\bar{E}_{i}^{\delta}} \\
\left|\Sigma_{p}^{i}\right| \lesssim 1 & \left|\Sigma_{q}^{i}\right| \lesssim \bar{E}_{i}^{-\frac{1}{2}}
\end{array}
$$

for some $\delta>0$. Here, the energies $\bar{E}_{i}$ are given by $\bar{E}_{i}=1+H_{f}\left(\bar{p}_{i}, \bar{q}_{i}\right)$.

Proof. This theorem gives a change of variables where the high-speed oscillations due to the presence of a breather located on the middle oscillator (that is the case where $E_{0}, E_{2} \ll E_{1}$ ) have been decoupled from the remaining degrees of freedom, leaving an effective "averaged out" dynamic. Recalling the formal calculation performed in Section 2, we see that when the energy $E$ is predominantly concentrated in the central oscillator, then the amplitudes of the oscillations for $p_{i}$ and $q_{i}, i=0,2$ scale to leading order like $E^{\frac{1}{k}-\frac{1}{2}}$ and $E^{\frac{3}{2 k}-1}$ respectively. This indicates that there are natural breakpoints at $k=2$ and $k=3 / 2$. When $k \geq 2$, the oscillations of both the $p_{i}$ and the $q_{i}$ are bounded as $E$ increases, so that they can be removed by a change of variables which is a bounded perturbation of the identity.

When $k<2$, the amplitude of the oscillations of the $p_{i}$ increases with $E$, but as long as $k \geq 3 / 2$, the amplitude of the $q_{i}$ does not. This growth will cause extra difficulties. If we consider $k<3 / 2$, both amplitudes would grow with $E$, leading to further complications. Since our goal is to outline the ideas without seeking the greatest generality, we resist the temptation to analyze all cases and restrict ourselves to the case $k>3 / 2$.

Before we proceed, let us compute the expressions $R$ and $\Sigma$ for a generic choice of $\Phi_{p}^{i}$ and $\Phi_{q}^{i}$. Applying Itôs formula to $\bar{p}_{i}$ and $\bar{q}_{i}$, we obtain

$$
\begin{aligned}
& R_{p}^{i}=V^{\prime}\left(q_{i}+\Phi_{q}^{i}\right)-V^{\prime}\left(q_{i}\right)+\Phi_{q}^{i}+\gamma_{i} \Phi_{p}^{i}+\mathcal{L} \Phi_{p}^{i}+q_{1} \\
& R_{q}^{i}=\mathcal{L} \Phi_{q}^{i}-\Phi_{p}^{i}, \quad \Sigma_{p}^{i}=\sigma_{i}\left(1+\partial_{p_{i}} \Phi_{p}^{i}\right), \quad \Sigma_{q}^{i}=\sigma_{i} \partial_{p_{i}} \Phi_{q}^{i} .
\end{aligned}
$$

Here and in the sequel we write $V(q)$ as a shorthand for $\frac{|q|^{2 k}}{2 k}$ and $\sigma_{i}$ as a shorthand for $\sqrt{2 \gamma_{i} T_{i}}$.

The case $k \geq 2$. The only "bad" term in the equations of motion for $\left(p_{i}, q_{i}\right)$ is the $q_{1}$ in the right hand side of the equation for $p_{i}$. The case $k \geq 2$ is much easier than the case $k<2$ since the system is more "rigid" in the later case. One can then simply take $\Phi_{q}^{i}=0$ and $\Phi_{p}^{i}=\Phi\left(p_{1}, q_{1}\right)$, where $\Phi$ is the centered solution to the Poisson equation

$$
X_{H_{f}} \Phi=\mathcal{R}(P, Q)-Q
$$

and $\mathcal{R}(P, Q)=Q \psi\left(H_{f}(P, Q)\right)$, where $\psi: \mathbf{R} \rightarrow[0,1]$ is a smooth function such that $\psi(x)=1$ for $|x| \leq 1$ and $\psi(x)=0$ for $|x| \geq 2$. Making this choice of $\Phi_{p}^{i}$ and $\Phi_{q}^{i}$ in (4.3) yields

$$
\begin{array}{ll}
\Sigma_{q}^{i}=0, & R_{q}^{i}=-\Phi\left(p_{1}, q_{1}\right), \\
\Sigma_{p}^{i}=\sigma_{i}, & R_{p}^{i}=\gamma_{i} \Phi\left(p_{1}, q_{1}\right)+\partial_{P} \Phi\left(p_{1}, q_{1}\right)\left(q_{0}+q_{2}-2 q_{1}\right)+\mathcal{R}\left(p_{1}, q_{1}\right)
\end{array}
$$

Since the function $\Phi$ scales like $H_{f}^{\frac{1}{k}-\frac{1}{2}}$ outside of a compact set, it can be checked easily that the bounds (4.2) hold, provided that $k \geq 2$. Note that in this case, $E_{i}$ and 
$\bar{E}_{i}$ are equivalent in the sense that $E_{1} \lesssim \bar{E}_{i} \lesssim E_{i}$ since $\Phi$ is bounded. Therefore all occurrences of $E_{j}$ in the bounds can be replaced by $\bar{E}_{j}$ at the cost of multiplicative constants.

The case $3 / 2<k<2$. We are now going to assume that $k<2$, which the more delicate case. Note that the second and third terms in $R_{p}^{i}$ above satisfy the bounds (4.2) (with $E_{j}$ instead of $\bar{E}_{j}$, but this problem will be dealt with later) provided that $Q \partial_{P} \Phi$ scales like $\hat{H}^{\theta}$ for some $\theta \leq 0$. This is the case when $k \geq \frac{3}{2}$, which is one of the reasons why we restrict ourselves to this case. Therefore, only the terms involving $\Phi$ scale worse then the desired bounds on the error terms and need to be eliminated. This motivates the introduction of the solution $\Phi^{(2)}$ to the Poisson equation

$$
X_{H_{f}} \Phi^{(2)}=\Phi .
$$

Note that $\Phi^{(2)}$ scales like $\hat{H}^{\frac{3}{2 k}-1}$ outside of a compact set, so that it is bounded if $k \geq \frac{3}{2}$. It would be tempting at this point to simply subtract $\gamma_{i} \Phi^{(2)}\left(p_{1}, q_{1}\right)$ to $p_{i}$ and add $\Phi^{(2)}\left(p_{1}, q_{1}\right)$ to $q_{i}$.

This would however introduce correction terms that grow faster than the bounds in (4.2). The trick is to realize that these correction terms are multiplied with terms that go to 0 as the energy of the middle oscillator becomes large. We therefore multiply $\Phi^{(2)}$ with a cutoff function that makes sure that this second round of correction is applied only when the energy of the middle oscillator is large compared to the energy of the boundary oscillators.

Let $\varphi: \mathbf{R}_{+} \rightarrow[0,1]$ be a smooth increasing function such that $\varphi(x)=0$ for $x \leq 1$ and $\varphi(x)=1$ for $x \geq 2$. Let furthermore $\alpha$ be an exponent to be determined later and set

$$
\begin{aligned}
& \Phi_{p}^{i}=\Phi\left(p_{1}, q_{1}\right)-\gamma_{i} \Phi^{(2)}\left(p_{1}, q_{1}\right), \\
& \Phi_{q}^{i}=\varphi\left(E_{1} / E_{i}^{\alpha}\right) \Phi^{(2)}\left(p_{1}, q_{1}\right),
\end{aligned}
$$

where we defined $E_{i}=1+H_{f}\left(p_{i}, q_{i}\right)$. In the sequel, we are going to use the shorthand $\varphi_{i}^{\alpha}=\varphi\left(E_{1} / E_{i}^{\alpha}\right)$ and we will omit the arguments of $\Phi, \Phi^{(2)}$ and $\varphi_{i}^{\alpha}$ in order to simplify notations. Before we turn to the verification of the bounds (4.2), we remark that since $k<2, E_{i}$ and $\bar{E}_{i}$ are not equivalent for $i=0,2$. Since $k \geq 3 / 2, E_{1}$ and $\bar{E}_{1}$ are however equivalent in the sense that $E_{1} \lesssim \bar{E}_{1} \lesssim E_{1}$. Since we wish to bound the remainder in terms of powers of $\bar{E}_{i}$ and not $E_{i}$, we are now going to show how these quantities are related. From the definitions of $\bar{p}_{i}$ and $\bar{q}_{i}$, we have for $i=0,2$ the estimate

$$
\left|\bar{E}_{i}-E_{i}\right| \lesssim 1+\left|p_{i} \Phi\right| \lesssim 1+\varepsilon p_{i}^{2}+\frac{1}{\varepsilon} \Phi^{2} \lesssim 1+\varepsilon E_{i}+\frac{1}{\varepsilon} E_{1}^{\frac{2}{k}-1} .
$$

By choosing $\varepsilon$ sufficiently small and moving the term $\varepsilon E_{i}$ to the left hand side, we thus obtain the two bounds

$$
E_{i} \lesssim \bar{E}_{i}+E_{1}^{\frac{2}{k}-1}, \quad \bar{E}_{i} \lesssim E_{i}+E_{1}^{\frac{2}{k}-1}
$$

Writing $g=E_{1} / E_{i}^{\alpha}$, and applying Itô's formula to $g$, we obtain

$$
\begin{gathered}
d g=E_{i}^{-\alpha} p_{1}\left(q_{0}+q_{2}-2 q_{1}\right) d t-\alpha E_{1} E_{i}^{-\alpha-1}\left(\frac{\sigma_{i}^{2}}{2}-\gamma_{i} p_{i}^{2}+q_{1}-q_{i}\right) d t \\
+\frac{\alpha(\alpha+1)}{2} E_{1} E_{i}^{-\alpha-2} \sigma_{i}^{2} p_{i}^{2} d t-\alpha E_{1} E_{i}^{-\alpha-1} \sigma_{i} p_{i} d w_{i}
\end{gathered}
$$




$$
=I_{1} d t+I_{2} d t+I_{3} d t+d M(t) .
$$

$M$ is a martingale since, on any finite time interval, it is easy to get control over the expected value of any power of the total energy. (Even a bound which grows exponentially with the length of the time interval is sufficient.) Bounding each of the three terms $I_{j}$ separately we obtain:

$$
\begin{aligned}
\left|I_{1}\right| \lesssim E_{i}^{-\alpha+\frac{1}{2 k}} E_{1}^{\frac{1}{2}}+E_{i}^{-\alpha} E_{2-i}^{\frac{1}{2 k}} E_{1}^{\frac{1}{2}}+E_{i}^{-\alpha} E_{1}^{\frac{1}{2}+\frac{1}{2 k}} \\
\left|I_{2}\right| \lesssim E_{1} E_{i}^{-\alpha}+E_{1}^{1+\frac{1}{2 k}} E_{i}^{-\alpha-1} \\
\left|I_{3}\right| \lesssim E_{1} E_{i}^{-\alpha-1} .
\end{aligned}
$$

Applying Itô's formula to $\varphi_{i}^{\alpha}$, we get

$$
\mathcal{L} \varphi_{i}^{\alpha}=\varphi^{\prime}\left(E_{1} / E_{i}^{\alpha}\right) \mathcal{L} g+\alpha^{2} \sigma_{i}^{2} \varphi^{\prime \prime}\left(E_{1} / E_{i}^{\alpha}\right) E_{1}^{2} E_{i}^{-2 \alpha-2} p_{i}^{2}=I_{4}+I_{5} .
$$

Note now that $\varphi^{\prime}$ and $\varphi^{\prime \prime}$ are zero outside the interval [1,2]. Therefore, we have $E_{1} \lesssim$ $E_{i}^{\alpha} \lesssim E_{1}$ on the support of these functions. This line of reasoning yields for $1 / k \leq$ $\alpha \leq 2 k$ the bounds:

$$
\begin{aligned}
& \left|I_{4}\right| \lesssim E_{i}^{\frac{1}{2}\left(\frac{1}{k}-\alpha\right)}+E_{i}^{-\frac{\alpha}{2}} E_{2-i}^{\frac{1}{2 k}}+E_{i}^{\frac{\alpha}{2}\left(\frac{1}{k}-1\right)}+1+E_{i}^{\frac{\alpha}{2 k}-1}+E_{i}^{-1} \lesssim E_{2-i}^{\frac{1}{2 k}}, \\
& \left|I_{5}\right| \lesssim E_{i}^{-1} \lesssim 1 .
\end{aligned}
$$

Collecting these estimates and taking into account the support of $\varphi^{\prime}$ and $\varphi^{\prime \prime}$ produces $\left|\mathcal{L} \varphi_{i}^{\alpha}\right| \lesssim\left(1+E_{2-i}^{\frac{1}{2 k}}\right) \mathbf{1}_{2 E_{i}^{\alpha} \geq E_{1} \geq E_{i}^{\alpha} .}$

Recall that, from the scalings given in Lemma 3.5 of the solutions to the Poisson equation (3.2) and the definitions (4.4) and (4.5) of $\Phi$ and $\Phi^{(2)}$, we have that $|\Phi| \lesssim$ $E_{1}^{\frac{1}{k}-\frac{1}{2}},\left|\partial_{P} \Phi\right| \lesssim E_{1}^{\frac{1}{k}-1},\left|\Phi^{(2)}\right| \lesssim E_{1}^{\frac{3}{2 k}-1},\left|\partial_{P} \Phi^{(2)}\right| \lesssim E_{1}^{\frac{3}{2 k}-\frac{3}{2}}$. We will also use the fact that, from the definition of $\varphi_{i}^{\alpha}$ combined with (4.7), one has both bounds

$$
E_{i}^{\alpha} \lesssim E_{1}, \quad \bar{E}_{i}^{\alpha} \lesssim E_{1},
$$

on the support of $\varphi_{i}^{\alpha}$. Furthermore, one has the bound $E_{1} \lesssim E_{i}^{\alpha}$ on the support of $1-\varphi_{i}^{\alpha}$ which, by (4.7), implies that one also has $E_{1} \lesssim \bar{E}_{i}^{\alpha}$ on the support of $1-\varphi_{i}^{\alpha}$.

Using the definitions of $\Phi_{p}^{i}$ and $\Phi_{q}^{i}$ given in (4.6), equation (4.3) yields

$$
R_{q}^{i}=-\Phi+\varphi_{i}^{\alpha} \mathcal{L} \Phi^{(2)}+\left(\mathcal{L} \varphi_{i}^{\alpha}\right) \Phi^{(2)}+\gamma_{i} \Phi^{(2)} .
$$

We now make use of the definitions of $\mathcal{L}$ and $\Phi^{(2)}$ to obtain

$$
\mathcal{L} \Phi^{(2)}=\Phi+\left(q_{0}+q_{2}-2 q_{1}\right) \partial_{P} \Phi^{(2)} .
$$

This allows us to obtain the following bounds for $R_{q}^{i}$ :

$$
\begin{aligned}
\left|R_{q}^{i}\right|= & \left|\left(\varphi_{i}^{\alpha}-1\right) \Phi+\varphi_{i}^{\alpha}\left(q_{0}+q_{2}-2 q_{1}\right) \partial_{P} \Phi^{(2)}+\left(\mathcal{L} \varphi_{i}^{\alpha}\right) \Phi^{(2)}+\gamma_{i} \Phi^{(2)}\right|, \\
\lesssim & \left(1-\varphi_{i}^{\alpha}\right) E_{1}^{\frac{1}{k}-\frac{1}{2}}+\varphi_{i}^{\alpha} E_{1}^{-\frac{3}{2}\left(1-\frac{1}{k}\right)}\left(E_{i}^{\frac{1}{2 k}}+E_{1}^{\frac{1}{2 k}}+E_{2-i}^{\frac{1}{2 k}}\right) \\
& +E_{2-i}^{\frac{1}{2 k}} E_{1}^{\frac{3}{2 k}-1} \mathbf{1}_{2 E_{i}^{\alpha} \geq E_{1} \geq E_{i}^{\alpha}+1} \\
= & I_{6}+I_{7}+I_{8}+1 .
\end{aligned}
$$


Our aim is to bound the terms $I_{6}, I_{7}, I_{8}$ in terms of the $\bar{E}_{j}$ 's instead of the $E_{j}$ 's. To do so, we now fix

$$
\alpha=\frac{3}{2} \text {. }
$$

Since $k \in(3 / 2,2), \alpha \in(1 / k, 2 k)$ which is the constraint that we had to impose earlier.

Since, as mentioned above, $E_{1} \lesssim \bar{E}_{i}^{\alpha}$ on the support of $1-\varphi_{i}^{\alpha}$, one has

$$
\left|I_{6}\right| \lesssim \bar{E}_{i}^{\frac{\alpha}{k}-\frac{\alpha}{2}}
$$

One can check that the choice $\alpha=3 / 2$ implies that there exists $\delta>0$ so that $\frac{\alpha}{k}-\frac{\alpha}{2} \leq$ $\frac{1}{2 k}-\delta$ (actually, one can set $\delta=1 / 12$ for the range of values of $k$ considered here). This shows that $\left|I_{6}\right| \lesssim \bar{E}_{i}^{\frac{1}{2 k}-\delta}$ as required.

To bound $I_{7}$, we make use of the fact that

$$
E_{i}^{\frac{1}{2 k}}+E_{2-i}^{\frac{1}{2 k}} \lesssim \bar{E}_{i}^{\frac{1}{2 k}}+E_{1}^{\frac{1}{k^{2}}-\frac{1}{2 k}}+\bar{E}_{2-i}^{\frac{1}{2 k}} \lesssim \bar{E}_{i}^{\frac{1}{2 k}}+E_{1}^{\frac{1}{2 k}}+\bar{E}_{2-i}^{\frac{1}{2 k}}
$$

so that, by virtue of (4.8),

$$
\begin{aligned}
\left|I_{7}\right| & \lesssim \varphi_{i}^{\alpha} E_{1}^{-\frac{3}{2}\left(1-\frac{1}{k}\right)}\left(\bar{E}_{i}^{\frac{1}{2 k}}+\bar{E}_{2-i}^{\frac{1}{2 k}}\right)+\varphi_{i}^{\alpha} E_{1}^{\frac{2}{k}-\frac{3}{2}} \\
& \lesssim \bar{E}_{i}^{-\delta}\left(\bar{E}_{i}^{\frac{1}{2 k}}+\bar{E}_{2-i}^{\frac{1}{2 k}}\right)+1
\end{aligned}
$$

We finally turn to $I_{8}$. We first remark that, by (4.7) and the fact that $\alpha\left(\frac{2}{k}-1\right)<1$, one has $\bar{E}_{i}^{\alpha} \lesssim E_{1} \lesssim \bar{E}_{i}^{\alpha}$ on the support of the indicator function $\mathbf{1}_{A}$ where $A=$ $\left\{2 E_{i}^{\alpha} \geq E_{1} \geq E_{i}^{\alpha}\right\}$. We thus obtain

$$
\begin{aligned}
\left|I_{8}\right| & \lesssim E_{2-i}^{\frac{1}{2 k}} \bar{E}_{i}^{\frac{3 \alpha}{2 k}-\alpha} \mathbf{1}_{A} \lesssim\left(\bar{E}_{2-i}^{\frac{1}{2 k}}+E_{1}^{\frac{1}{2 k}\left(\frac{2}{k}-1\right)}\right) \bar{E}_{i}^{\frac{3 \alpha}{2 k}-\alpha} \mathbf{1}_{A} \\
& \lesssim\left(\bar{E}_{2-i}^{\frac{1}{2 k}}+\bar{E}_{i}^{\frac{\alpha}{2 k}\left(\frac{2}{k}-1\right)}\right) \bar{E}_{i}^{\frac{3 \alpha}{2 k}-\alpha} \lesssim \bar{E}_{2-i}^{\frac{1}{2 k}} \bar{E}_{i}^{-\delta}+\bar{E}_{i}^{\alpha \frac{1+k-k^{2}}{k^{2}}}
\end{aligned}
$$

One can check that, for $\alpha=3 / 2$ and the range of $k$ 's of interest, the exponent of the last term is strictly smaller than $1 / 2 k$. Therefore, $\left|I_{8}\right| \lesssim \bar{E}_{i}^{-\delta}\left(\bar{E}_{i}^{\frac{1}{2 k}}+\bar{E}_{2-i}^{\frac{1}{2 k}}\right)$ as required, choosing $\delta$ smaller if necessary. Collecting all of these bounds shows that $R_{q}^{i}$ does indeed satisfy the bound advertised in (4.2).

Turning to $R_{p}^{i},(4.3)$ yields

$$
\begin{aligned}
\left|R_{p}^{i}\right|=\mid \mathcal{R}\left(q_{1}\right)+\left(q_{0}+q_{2}-2 q_{1}\right)\left(\partial_{P} \Phi-\gamma_{i} \partial_{P} \Phi^{(2)}\right)-\left(\gamma_{i}^{2}-\varphi_{i}^{\alpha}\right) \Phi^{(2)} \\
\quad+V^{\prime}\left(q_{i}+\varphi_{i}^{\alpha} \Phi^{(2)}\right)-V^{\prime}\left(q_{i}\right) \mid \\
\lesssim 1+\left(\bar{E}_{0}^{\frac{1}{2 k}}+\bar{E}_{2}^{\frac{1}{2 k}}+E_{1}^{\frac{1}{2 k}}\right) E_{1}^{\frac{1}{k}-1}+1+\left|V^{\prime}\left(q_{i}+\varphi_{i}^{\alpha} \Phi^{(2)}\right)-V^{\prime}\left(q_{i}\right)\right| .
\end{aligned}
$$

Here, we made use of (4.9) and of (4.7). Since, for $k \geq 3 / 2, \Phi^{(2)}$ is a bounded function, one has the further bound

$$
\begin{aligned}
\left|V^{\prime}\left(q_{i}+\varphi_{i}^{\alpha} \Phi^{(2)}\right)-V^{\prime}\left(q_{i}\right)\right| & =\left|V^{\prime}\left(\bar{q}_{i}\right)-V^{\prime}\left(\bar{q}_{i}-\varphi_{i}^{\alpha} \Phi^{(2)}\right)\right| \\
& \lesssim\left(1+\left|\bar{q}_{i}\right|\right)^{2 k-2}\left|\Phi^{(2)}\right| \lesssim \bar{E}_{i}^{1-\frac{1}{k}} \lesssim \bar{E}_{i}^{\frac{1}{2}-\delta},
\end{aligned}
$$

for $\delta$ sufficiently small. Collecting these bounds and using the fact that $k>3 / 2$, we obtain

$$
\left|R_{p}^{i}\right| \lesssim \bar{E}_{0}^{\frac{1}{2 k}}+\bar{E}_{2}^{\frac{1}{2 k}}+\bar{E}_{i}^{\frac{1}{2}-\delta} \lesssim \bar{E}_{i}^{\frac{1}{2}-\delta}+\bar{E}_{2-i}^{\frac{1}{2}-\delta}
$$


which is indeed of the form (4.2).

Lastly, the $\Sigma$-terms can be bounded by

$$
\begin{aligned}
\left|\Sigma_{q}^{i}\right| & =\left|\alpha \sigma_{i} \varphi^{\prime}\left(E_{1} / E_{i}^{\alpha}\right) \Phi^{(2)} E_{1} E_{i}^{-\alpha-1} p_{i}\right| \lesssim \bar{E}_{i}^{-\frac{1}{2}}, \\
\Sigma_{p}^{i} & =\sigma_{i},
\end{aligned}
$$

where we made use of the fact that, like in the bound of $I_{8}, E_{1}, E_{1}^{\alpha}$ and $\bar{E}_{i}^{\alpha}$ are equivalent on the support of $\varphi^{\prime}\left(E_{1} / E_{i}^{\alpha}\right)$. This concludes the proof of Theorem 4.1.

In the case $k>2$, it will be useful in the sequel to have a better approximation of the dynamics that yields smaller error terms in the regime where most of the energy is located in the middle oscillator:

Theorem 4.2 Assume that $k>2$. There exist smooth functions $\Phi_{p}^{i}$ and $\Phi_{q}^{i}$ depending on $\left(p_{i}, q_{i}, p_{1}, q_{1}\right)$ such that if we make the change of variables $\bar{p}_{i}=p_{i}+\Phi_{p}^{i}$ and $\bar{q}_{i}=q_{i}+\Phi_{q}^{i}($ for $i=0,2)$, the equations of motion for $\left(\bar{p}_{i}, \bar{q}_{i}\right)$ are given by

$$
\begin{aligned}
d \bar{q}_{i} & =\bar{p}_{i} d t+R_{q}^{i} d t \\
d \bar{p}_{i} & =-\bar{q}_{i}\left|\bar{q}_{i}\right|^{2 k-2} d t-\bar{q}_{i} d t-\gamma_{i} \bar{p}_{i} d t+R_{p}^{i} d t+\sigma_{i} d w_{i}
\end{aligned}
$$

for some adapted processes $R_{p}^{i}$ and $R_{q}^{i}$. Furthermore, the error terms $R$ satisfy the bounds

$$
\left|R_{p}^{i}\right| \lesssim\left(E_{0}+E_{2}\right)^{2} H^{\frac{3}{2 k}-1} \quad\left|R_{q}^{i}\right| \lesssim\left(E_{0}+E_{2}\right) H^{\frac{3}{2 k}-1} .
$$

Here, the energies $E_{i}$ are given as before by $E_{i}=1+H_{f}\left(\bar{p}_{i}, \bar{q}_{i}\right)$ and $H$ is the total Hamiltonian of our system.

Proof. Following the proof of Theorem 4.1, we set as in (4.6)

$$
\begin{aligned}
& \Phi_{p}^{i}=\Phi\left(p_{1}, q_{1}\right)-\gamma_{i} \Phi^{(2)}\left(p_{1}, q_{1}\right), \\
& \Phi_{q}^{i}=\Phi^{(2)}\left(p_{1}, q_{1}\right) .
\end{aligned}
$$

This yields for $R_{p}^{i}$ and $R_{q}^{i}$ the expressions

$$
\begin{aligned}
R_{q}^{i}= & \left(q_{0}+q_{2}-2 q_{1}\right) \partial_{P} \Phi^{(2)}+\gamma_{i} \Phi^{(2)} \\
R_{p}^{i}= & \mathcal{R}\left(q_{1}\right)+\left(q_{0}+q_{2}-2 q_{1}\right)\left(\partial_{P} \Phi-\gamma_{i} \partial_{P} \Phi^{(2)}\right)-\left(\gamma_{i}^{2}-1\right) \Phi^{(2)} \\
& \quad+V^{\prime}\left(q_{i}+\Phi^{(2)}\right)-V^{\prime}\left(q_{i}\right) .
\end{aligned}
$$

The desired bounds now follow from Lemma 4.3 below, together with the fact that both $\Phi^{(2)}$ and $Q \partial_{P} \Phi$ scale like $H_{f}^{\frac{3}{2 k}-1}$.

Lemma 4.3 For every $\alpha>0$, the bound

$$
x^{-\alpha} \leq(x+y)^{-\alpha} \max \left\{2^{\alpha}, y^{\alpha}\right\}
$$

holds for every $x, y \geq 1$.

Proof. If $x \geq y$, then $x^{-\alpha} \leq 2^{\alpha}(2 x)^{-\alpha} \leq 2^{\alpha}(x+y)^{-\alpha}$. If on the other hand $x \leq y$, then $x^{-\alpha} \leq 1 \leq y^{\alpha}(x+y)^{-\alpha}$. 


\section{Existence of an invariant measure}

\subsection{General strategy}

To prove the existence of an invariant measure, our aim is to construct a type of Lyapunov function $\mathcal{V}(p, q)$ such that $\mathcal{V}(p, q) \rightarrow \infty$ as $|(p, q)| \rightarrow \infty$ and $\mathcal{L} \mathcal{V}(p, q) \rightarrow-\infty$ as $|(p, q)| \rightarrow \infty$. Given such a function, the existence of an invariant measure will follow from the following proposition which is a variant of the classical Kryloff-Bogoliouboff construction [KB37, Has80, p. 52]:

Proposition 5.1 Consider an SDE on $\mathbf{R}^{n}$ with smooth coefficients and denote its generator by $\mathcal{L}$. Assume that the $S D E$ has global solutions and generates a Feller semigroup. If there exists a smooth function $\mathcal{V}: \mathbf{R}^{n} \rightarrow[0, \infty)$ such that the level sets $\{x: \mathcal{L V}(x) \geq C\}$ are compact for every $C$, then the $S D E$ possesses an invariant probability measure $\mu$. Furthermore, the function $\mathcal{L} \mathcal{V}$ is integrable with respect to $\mu$ and $\int \mathcal{L V}(x) \mu(d x)=0$.

We will construct the function $\mathcal{V}$ in steps by analyzing the dynamic in the limit of various energies being large and then draw inspiration from the structure of these limiting regimes to construct $\mathcal{V}$. Operationally, we will make an initial guess for $\mathcal{V}$ and then augment it by a series of correction terms.

Proof of Proposition 5.1. Denote by $x_{t}$ the solution to the SDE starting at some (deterministic) initial condition $x_{0}$. Applying Itô's formula to $\mathcal{V}\left(x_{t}\right)$, we get

$$
d \mathcal{V}\left(x_{t}\right)=(\mathcal{L} \mathcal{V})\left(x_{t}\right) d s+d M(t),
$$

for some continuous local martingale $M$. Therefore, there exists an increasing sequence $\tau_{N}$ of stopping times converging to $+\infty$ such that $M\left(t \wedge \tau_{N}\right)$ are martingales.

$$
\mathbf{E} \mathcal{V}\left(x_{t \wedge \tau_{N}}\right)-\mathcal{V}\left(x_{0}\right)-\mathbf{E} \int_{0}^{t \wedge \tau_{N}}(\mathcal{L} \mathcal{V})\left(x_{s}\right) d s=0 .
$$

Since $\mathcal{V}$ is positive this shows that, for every $K>0$,

$$
\mathbf{E} \int_{0}^{t \wedge \tau_{N}}\left(K-(\mathcal{L} \mathcal{V})\left(x_{s}\right)\right) d s \leq \mathcal{V}\left(x_{0}\right)+K t .
$$

Taking $K$ large enough so that $K-\mathcal{L V} \geq 0$, we can apply the monotone convergence theorem to take the limit $N \rightarrow \infty$ and obtain

$$
-\mathbf{E} \int_{0}^{t}(\mathcal{L} \mathcal{V})\left(x_{s}\right) d s \leq \mathcal{V}\left(x_{0}\right) .
$$

Now, by assumption the sets $A_{R}=\{x:-\mathcal{L V}(x) \leq R\}$ are compact for all $R$. In particular, this implies that there exist a $K>0$ so that $-\mathcal{L V}(x)+K \geq 0$ for all $x$. Now observe that for $R>-K$

$$
\begin{aligned}
\frac{1}{t} \int_{0}^{t} \mathbf{P}\left(x_{s} \notin A_{R}\right) d s & =\frac{1}{t} \int_{0}^{t} \mathbf{P}\left(-\mathcal{L} \mathcal{V}\left(x_{s}\right)+K>R+K\right) d s \\
& \leq \frac{1}{t} \int_{0}^{t} \frac{K-\mathbf{E} \mathcal{L} \mathcal{V}\left(x_{s}\right)}{R+K} d s \leq \frac{K+\mathcal{V}\left(x_{0}\right)}{K+R} .
\end{aligned}
$$


Therefore, the sequence of measures $\mu_{t}$ defined for measurable sets $A$ by

$$
\mu_{t}(A)=\frac{1}{t} \int_{0}^{t} \mathbf{P}_{x_{0}}\left(x_{s} \in A\right) d s
$$

is tight. Hence the Kryloff-Bogoliouboff construction [KB37, Has80, p. 52] guarantees the existence of an invariant measure. The last statement then follows from Lebesgue's dominated convergence theorem.

Remark 5.2 We will actually be able to construct a positive smooth function $\mathcal{V}$ with compact level sets that has the property that

$$
\mathcal{L} \mathcal{V} \leq C_{1}-C_{2} \mathcal{V}^{\alpha}
$$

for some positive constants $C_{i}$ and some (typically quite small) $\alpha \in(0,1]$. In this case, it is known [DFG06] that one does not only have the existence of an invariant measure, but the transition probabilities converge towards it at rate $\mathcal{O}\left(t^{-\alpha /(1-\alpha)}\right)$ in the total variation distance. We believe that this convergence actually takes place at a much faster rate, but such a statement is beyond our reach at the moment. See also [Ver97] for related results on subexponential mixing for SDEs.

\subsection{Construction of the Lyapunov function}

Recall the change of variables $(p, q) \mapsto(\bar{p}, \bar{q})$ from Theorem 4.1 that leads to an effective decoupled dynamic for the outside oscillators. In order to construct the Lyapunov function $\mathcal{V}$, we proceed in two steps:

1. We gain good control over the dissipation of the energy stored in the outside oscillators. This will be the content of Proposition 5.3.

2. We use this in order to get control over the dissipation of the total energy of the system in Theorem 5.6.

Proposition 5.3 There is a function $\mathcal{U}_{0}$ equivalent to $H_{f}\left(\bar{p}_{0}, \bar{q}_{0}\right)+H_{f}\left(\bar{p}_{2}, \bar{q}_{2}\right)$ and such that, for every $m>0$, there exist constants $C_{m}>0$ and $c_{m}>0$ such that $\mathcal{L U}_{0}^{m} \leq$ $C_{m}-c_{m} \mathcal{U}_{0}^{m}$.

Proof. Inspired by (4.1), we define an effective Hamiltonian $H_{0}$ by

$$
H_{0}(p, q)=\frac{p^{2}}{2}+\frac{|q|^{2 k}}{2 k}+\frac{q^{2}}{2}+1 .
$$

(Note that $H_{0}$ is equivalent to $H_{f}$.) We set

$$
\mathcal{U}_{0}=H_{0}\left(\bar{p}_{0}, \bar{q}_{0}\right)+H_{0}\left(\bar{p}_{2}, \bar{q}_{2}\right)+\gamma\left(\bar{p}_{0} \bar{q}_{0}+\bar{p}_{2} \bar{q}_{2}\right)
$$

for some constant $\gamma$ to be determined later. If $\gamma$ is sufficiently small and since $k \geq 1$, this function is indeed equivalent to $\bar{E}_{0}+\bar{E}_{2}=H_{f}\left(\bar{p}_{0}, \bar{q}_{0}\right)+H_{f}\left(\bar{p}_{2}, \bar{q}_{2}\right)$. Applying Itô's formula to it, we get from (4.1) that

$$
\begin{aligned}
d \mathcal{U}_{0}= & \sum_{i=0,2}\left(\left(\gamma-\gamma_{i}\right) \bar{p}_{i}^{2}-\gamma\left(\left|\bar{q}_{i}\right|^{2 k}+\left|\bar{q}_{i}\right|^{2}\right)-\gamma \gamma_{i} \bar{p}_{i} \bar{q}_{i}+\left(\bar{p}_{i}+\gamma \bar{q}_{i}\right) R_{p}^{i}\right. \\
& \left.\quad+\left(\left|\bar{q}_{i}\right|^{2 k-2} \bar{q}_{i}+\gamma \bar{p}_{i}\right) R_{q}^{i}+\left(k-\frac{1}{2}\right)\left|\bar{q}_{i}\right|^{2 k-2}\left|\Sigma_{q}^{i}\right|^{2}+\frac{1}{2}\left|\Sigma_{p}^{i}\right|^{2}+\gamma \Sigma_{p}^{i} \Sigma_{q}^{i}\right) d t
\end{aligned}
$$




$$
+\sum_{i=0,2}\left(\left(\bar{p}_{i}+\gamma \bar{q}_{i}\right) \Sigma_{p}^{i}+\left(\left|\bar{q}_{i}\right|^{2 k-2} \bar{q}_{i}+\bar{q}_{i}+\gamma \bar{p}_{i}\right) \Sigma_{q}^{i}\right) d w_{i} .
$$

Fixing $\gamma=\frac{1}{2} \min \left\{1, \gamma_{0}, \gamma_{2}\right\}$, we obtain for some constant $C$

$$
\begin{aligned}
d \mathcal{U}_{0} \leq & -2 \gamma \mathcal{U}_{0} d t+C \sum_{i=0,2}\left(\left|\bar{p}_{i} \bar{q}_{i}\right|+\left|\bar{p}_{i}+\gamma \bar{q}_{i}\right|\left|R_{p}^{i}\right|\right. \\
& \left.+\left(\left|\bar{q}_{i}\right|^{2 k-1}+\left|\bar{p}_{i}\right|\right)\left|R_{q}^{i}\right|+\left(1+\left|\bar{q}_{i}\right|^{2 k-2}\right)\left|\Sigma_{q}^{i}\right|^{2}+\left|\Sigma_{p}^{i}\right|^{2}\right) d t+d M(t) .
\end{aligned}
$$

Here, $M$ is a continuous Martingale with quadratic variation bounded by

$$
\frac{d\langle M\rangle(t)}{d t} \leq C \sum_{i=0,2}\left(\left(\left|\bar{p}_{i}\right|^{2}+\left|\bar{q}_{i}\right|^{2}\right)\left|\Sigma_{p}^{i}\right|^{2}+\left(1+\left|\bar{q}_{i}\right|^{4 k-2}+\left|\bar{p}_{i}\right|^{2}\right)\left|\Sigma_{q}^{i}\right|^{2}\right) .
$$

Using the notation and the bounds of Theorem 4.1, we have

$$
\begin{aligned}
\left|\bar{p}_{i} \bar{q}_{i}\right| & \lesssim \bar{E}_{i}^{\frac{1}{2}+\frac{1}{2 k}}, & \left|\bar{p}_{i}+\gamma \bar{q}_{i}\right|\left|R_{p}^{i}\right| & \lesssim \bar{E}_{i}^{1-\delta}, \\
\left(\left|\bar{q}_{i}\right|^{2 k-1}+\left|\bar{p}_{i}\right|\right)\left|R_{q}^{i}\right| & \lesssim \bar{E}_{i}^{1-\delta}+\bar{E}_{2-i}^{1-\delta}, & \left(1+\left|\bar{q}_{i}\right|^{2 k-2}\right)\left|\Sigma_{q}^{i}\right|^{2} & \lesssim \bar{E}_{i}^{\frac{1}{2}-\frac{1}{k}} .
\end{aligned}
$$

Since all the powers appearing on the right hand sides of these bounds are strictly less than 1 , we have shown that

$$
\mathcal{L U}_{0} \leq C-\gamma \mathcal{U}_{0}
$$

for some (different) constant $C$.

In order to bound the quadratic variation of $M$, note that one has

$$
\left(\left|\bar{p}_{i}\right|^{2}+\left|\bar{q}_{i}\right|^{2}\right)\left|\Sigma_{p}^{i}\right|^{2} \lesssim \bar{E}_{i}, \quad\left(1+\left|\bar{q}_{i}\right|^{4 k-2}+\left|\bar{p}_{i}\right|^{2}\right)\left|\Sigma_{q}^{i}\right|^{2} \lesssim \bar{E}_{i}^{1-\frac{1}{k}}
$$

In particular, one has for some positive constant $C$

$$
\langle M\rangle(t) \leq C \int_{0}^{t}\left(1+\mathcal{U}_{0}(s)\right) d s .
$$

The claim now follows by applying Itô's formula to $\mathcal{U}_{0}^{m}$.

Remark 5.4 Observe that one could also apply Itô's formula to $\exp \left(\theta \mathcal{U}_{0}\right)$ for sufficiently small $\theta$ and obtain $\mathcal{L} \exp \left(\theta \mathcal{U}_{0}\right) \leq C-\alpha \exp \left(\theta \mathcal{U}_{0}\right)$.

Remark 5.5 If $k \geq 2$, then $\mathcal{U}_{0}$ is also equivalent to $H_{f}\left(p_{0}, q_{0}\right)+H_{f}\left(p_{2}, q_{2}\right)$. This is not the case when $k<2$.

We are now ready to prove the main theorem of this section. Recall that $H$ is the total Hamiltonian defined in (1.4).

Theorem 5.6 Consider the equations of motion (2.1) with $k>3 / 2$. Then there exists a function $\mathcal{V}$ and constants $c, C>0$ such that $\mathcal{V} \geq c H^{\alpha}-C$ and such that $\mathcal{L} \mathcal{V} \leq$ $C-c H^{\alpha^{\prime}}$ for some exponents $\alpha$ and $\alpha^{\prime}$. 
Proof. The idea is to work "modulo powers of $\mathcal{U}_{0}$." Assume that we can find a function $\mathcal{U}_{1}$ such that

$$
\mathcal{U}_{1} \geq c H^{\alpha}-C \mathcal{U}_{0}^{N}, \quad \mathcal{L U}_{1} \leq-c H^{\alpha^{\prime}}+C \mathcal{U}_{0}^{N^{\prime}},
$$

for some positive exponents $\alpha, \alpha^{\prime}$ and some (possibly very large) exponents $N, N^{\prime}$. We claim that it then suffices to take $\mathcal{V}=\mathcal{U}_{1}+\mathcal{U}_{0}^{N+N^{\prime}+1}$. Note that

$$
\mathcal{V} \geq c H^{\alpha}-C \mathcal{U}_{0}^{N}+\mathcal{U}_{0}^{N+N^{\prime}+1} \geq c H^{\alpha}-C^{\prime},
$$

for some constant $C^{\prime}$, and so $\mathcal{V}$ grows at infinity (has compact level sets). It then follows from Proposition 5.3 and from (5.2) that

$$
\mathcal{L} \mathcal{V} \leq-c H^{\alpha^{\prime}}+\mathrm{CU}_{0}^{N^{\prime}}+C_{N+N^{\prime}+1}-c_{N+N^{\prime}+1} \mathcal{U}_{0}^{N+N^{\prime}+1} \leq C^{\prime \prime}-c H^{\alpha^{\prime}},
$$

for some constant $C^{\prime \prime}$, which is the desired bound. It therefore remains to construct a function $\mathcal{U}_{1}$ satisfying (5.2).

The starting point for the construction of $\mathcal{U}_{1}$ is to apply Itô's formula to $H^{n}$. Since $d H=\sum_{i=0,2}\left(-\gamma_{i} p_{i} d t+\frac{1}{2} \sigma_{i}^{2} d t+p_{i} \sigma_{i} d w_{i}\right)$, we have

$$
d H^{n}=n H^{n-2} \sum_{i=0,2}\left(H\left(\frac{\sigma_{i}^{2}}{2}-\gamma_{i} p_{i}^{2}\right) d t+\frac{1}{2}(n-1) \sigma_{i}^{2} p_{i}^{2} d t+H \sigma_{i} p_{i} d w_{i}\right) .
$$

At this stage, we have to distinguish two cases, as in the proof of Theorem 4.1:

- If $3 / 2<k<2$, then the motion of the interior oscillator induces large (i.e. going to infinity with the energy of the middle oscillator) fluctuations in the values of $p_{0}$ and $p_{2}$. Therefore, there will always be energy dissipation.

- If $k \geq 2$, then the motion of the interior oscillator induces bounded (or small) fluctuations in the values of $p_{0}$ and $p_{2}$. In this case, one can subtract a compensator so that only fluctuations remain. There will be a few error terms that seem to be larger than the dominant one, but they can hopefully just be eliminated order by order.

The case $3 / 2<k<2$ : In contrast to the arguments in the proof of Theorem 4.1, this is the "easy" case for this part of the proof. It follows from the proof of Theorem 4.1 that in this case $\bar{p}_{i}^{2}$ is equivalent to $\hat{p}_{i}^{2}$ with $\hat{p}_{i}=p_{i}-\Phi\left(p_{1}, q_{1}\right)$. Since $2 \gamma_{i} \hat{p}_{i} \Phi \leq$ $\frac{1}{2} \gamma_{i} \Phi^{2}+2 \gamma_{i} \hat{p}_{i}^{2}$, this allows us to obtain for $\mathcal{L} H^{n}$ the bound

$$
\mathcal{L} H^{n} \leq n H^{n-1}\left(C_{n}+\gamma_{0} \hat{p}_{0}^{2}+\gamma_{2} \hat{p}_{2}^{2}-\frac{1}{2}\left(\gamma_{0}+\gamma_{2}\right) \Phi^{2}\right),
$$

for some positive constant $C_{n}$. This in turn implies that

$$
\mathcal{L} H^{n} \leq H^{n-1}\left(C_{1}+C_{2} \mathcal{U}_{0}-\frac{n}{2}\left(\gamma_{0}+\gamma_{2}\right) \Phi^{2}\right),
$$

for some constants $C_{i}$. Observe that one can use a similar calculation to obtain the bound

$$
\left|\mathcal{L} H^{n}\right| \leq H^{n-1}\left(C_{1}+C_{2} \mathcal{U}_{0}+C_{3} \Phi^{2}\right),
$$

for some possibly different constants $C_{i}$.

Note that $\Phi^{2}$ scales like $H_{f}^{\frac{2}{k}-1}$ which goes to infinity at high energies. We can thus find a positive constant $\kappa_{k}$ (which was introduced in (2.8)) and a function $\mathcal{R}^{\prime}$ such that 
$\Phi^{2}-\kappa_{k} H_{f}^{\frac{2}{k}-1}+\mathcal{R}^{\prime}\left(H_{f}\right)$ averages out to 0 in the sense of Proposition 3.7. We define $\Psi$ to be the solution to the Poisson equation

$$
X_{H_{f}} \Psi=\Phi^{2}-\kappa_{k} H_{f}^{\frac{2}{k}-1}+\mathcal{R}^{\prime}\left(H_{f}\right) .
$$

This function is smooth since $\mathcal{R}^{\prime}$ is precisely such that $\Psi=0$ in a neighborhood of the origin. The function $\Psi$ then scales like $H_{f}^{\frac{5}{2 k}-\frac{3}{2}}$. In particular, $\Psi$ and $Q \partial_{P} \Psi$ are bounded.

With all these preliminaries done, we define

$$
\mathcal{U}_{1}=H^{n}+\frac{n}{2}\left(\gamma_{0}+\gamma_{2}\right) H^{n-1} \Psi .
$$

Applying Itô's formula to $\mathcal{U}_{1}$ and using (5.4), (5.5), and (5.6), we obtain

$$
\begin{aligned}
\mathcal{L U}_{1} \leq & C H^{n-1}\left(1+\mathcal{U}_{0}\right)+C|\Psi| H^{n-2}\left(1+\mathcal{U}_{0}+\Phi^{2}\right) \\
& +\frac{n}{2}\left(\gamma_{0}+\gamma_{2}\right) H^{n-1}\left(-\kappa_{k} E_{1}^{\frac{2}{k}-1}+\left(q_{0}+q_{2}-2 q_{1}\right) \partial_{P} \Psi\right) .
\end{aligned}
$$

Since $|\Psi| \Phi^{2} \lesssim H$ and $\left|\left(q_{0}+q_{2}-2 q_{1}\right) \partial_{P} \Psi\right| \lesssim \mathcal{U}_{0}$, we have

$$
\mathcal{L} \mathcal{U}_{1} \leq-\frac{\kappa_{k} n}{2}\left(\gamma_{0}+\gamma_{2}\right) H^{n-1} \kappa_{k} E_{1}^{\frac{2}{k}-1}+C H^{n-1} \mathcal{U}_{0},
$$

for some constant $C$.

We can check that $\mathcal{U}_{1}$ satisfies the first bound in (5.2), since $\Psi$ grows slower than $H$ and $\mathcal{U}_{0}$ is bounded from below by a positive constant. However, it is not clear a priori from this expression that it $\mathcal{U}_{1}$ satisfies the second bound in (5.2).

One can check that

$$
\mathcal{U}_{0} \geq c\left(E_{0}+E_{2}\right)-C \Phi^{2},
$$

for some positive constants $c$ and $C$. Furthermore, $\Phi^{2}$ scales like $H_{f}^{\frac{2}{k}-1}$ which is strictly smaller than $H_{f}$, so that

$$
\mathcal{U}_{0}+E_{1} \geq c H .
$$

In particular, there exists a constant $C$ such that

$$
H^{\frac{2}{k}-1} \leq C\left(E_{1}^{\frac{2}{k}-1}+\mathcal{U}_{0}^{\frac{2}{k}-1}\right) .
$$

Inserting this into (5.7), we obtain

$$
\mathcal{L} \mathcal{U}_{1} \leq-c H^{n+\frac{2}{k}-2}+C H^{n-1} \mathcal{U}_{0},
$$

for some constants $c$ and $C$. Since $\frac{2}{k}-1>0$, this shows the existence of a (sufficiently large) power $N$ such that

$$
\mathcal{L} \mathcal{U}_{1} \leq-c H^{n+\frac{2}{k}-2}+C \mathcal{U}_{0}^{N},
$$

which is the desired bound.

The case $k \geq 2$ : The reasoning above only worked for $k<2$ since in this case one has $n+\frac{2}{k}-2>n-1$. When $k \geq 2$ the situation is slightly more delicate. Here, as before, the energy dissipation mechanism comes from fluctuations of $p_{0}^{2}$ and $p_{2}^{2}$ around their mean. However, the amplitude of these oscillations now decreases as the energy stored 
in the middle oscillator increases, so that we have to be more careful. In particular, $p_{i}^{2}$ can no longer be treated as a perturbation with respect to $\Phi^{2}$, as we did in the previous case.

The expression in (5.3) suggests that, in order to extract these small fluctuations, we should compensate $H^{n}$ by subtracting

$$
\Xi=n H^{n-1}\left[H_{0}\left(\bar{p}_{0}, \bar{q}_{0}\right)+H_{0}\left(\bar{p}_{2}, \bar{q}_{2}\right)\right],
$$

where the "bar" variables are as in Theorem 4.2. Note that here, and anywhere from this point on, we use the variables $\left(\bar{p}_{i}, \bar{q}_{i}\right)$ from Theorem 4.2 and not from Theorem 4.1. This is because we require a sufficiently good effective dynamics so that the error terms are small with respect to $\Phi^{2}$. The bounds obtained in Theorem 4.1 are not sufficiently small for that. Setting as before $\bar{E}_{i}$ as a shorthand for $H_{f}\left(\bar{p}_{i}, \bar{q}_{i}\right)$, one has

$$
\begin{aligned}
\mathcal{L} \Xi= & n H^{n-2} \sum_{i=0,2}\left(H\left(\frac{\sigma_{i}^{2}}{2}-\gamma_{i} \bar{p}_{i}^{2}\right)+(n-1) \bar{E}_{i}\left(\frac{\sigma_{i}^{2}}{2}-\gamma_{i} p_{i}^{2}\right)\right. \\
& +\frac{1}{2}(n-1)(n-2) \bar{E}_{i} H^{-1} \sigma_{i}^{2} p_{i}^{2}+(n-1) \sigma_{i}^{2} p_{i} \bar{p}_{i} \\
& \left.+H\left(\bar{p}_{i} R_{p}^{i}+\bar{q}_{i}\left(\left|\bar{q}_{i}\right|^{2 k-2}+1\right) R_{q}^{i}\right)\right)
\end{aligned}
$$

The important term in this expression is $n H^{n-1}\left(\frac{\sigma_{0}^{2}+\sigma_{2}^{2}}{2}-\gamma_{0} \bar{p}_{0}^{2}-\gamma_{2} \bar{p}_{2}^{2}\right)$, all the other terms will be treated as perturbations. Setting $\mathcal{U}_{1}^{0}=H^{n}-\Xi$, we obtain

$$
\mathcal{L} \mathcal{U}_{1}^{0}=-n H^{n-1} \sum_{i=0,2} \gamma_{i}\left(p_{i}^{2}-\bar{p}_{i}^{2}\right)+\mathcal{E} .
$$

with

$$
\begin{aligned}
\mathcal{E}= & n H^{n-2} \sum_{i=0,2}\left(-(n-1) \bar{E}_{i}\left(\frac{\sigma_{i}^{2}}{2}-\gamma_{i} p_{i}^{2}\right)-H\left(\bar{p}_{i} R_{p}^{i}+\bar{q}_{i}\left(\left|\bar{q}_{i}\right|^{2 k-2}+1\right) R_{q}^{i}\right)\right. \\
& \left.-\frac{1}{2}(n-1) \sigma_{i}^{2}\left((n-2) \bar{E}_{i} H^{-1} p_{i}^{2}-2 p_{i} \bar{p}_{i}+p_{i}^{2}\right)\right) .
\end{aligned}
$$

We can check that one has $|\mathcal{E}| \lesssim H^{n-2+\frac{3}{2 k}} \mathcal{U}_{0}^{2}$ so that, for every $\delta>0$, there exist constants $C$ and $N$ such that the error term $\mathcal{E}$ is bounded by $H^{n+\frac{3}{2 k}-2+\delta}+C \mathcal{U}_{0}^{N}$.

At this stage, we use the explicit expression for $\bar{p}_{i}$ to rewrite this bound as

$$
\mathcal{L} \mathcal{U}_{1}^{0} \leq-n H^{n-1} \sum_{i=0,2} \gamma_{i}\left(\Phi^{2}+\gamma_{i}^{2}\left(\Phi^{(2)}\right)^{2}-2 \gamma_{i} \Phi \Phi^{(2)}+2 \bar{p}_{i}\left(\gamma_{i} \Phi^{(2)}-\Phi\right)\right)+\mathcal{E} .
$$

This puts us now in a situation similar to (5.4), with the difference that, up to powers of $\mathcal{U}_{0}$, the error term $\mathcal{E}$ is of order $H^{n-2+\frac{3}{2 k}+\delta}$ instead of being of order $H^{n-1}$. Since $\Phi^{2}$ is larger than $H_{f}^{\frac{3}{2 k}-1}$, this is the feature that will allow us to obtain the required bound.

Since $\Phi^{(2)}$ scales like $H_{f}^{\frac{3}{2 k}-1}$, we obtain in the same way as in the proof of Theorem 4.2 that $\left|\Phi^{(2)}\right| \lesssim H^{\frac{3}{2 k}-1} \mathcal{U}_{0}^{1-\frac{3}{2 k}}$. This shows that

$$
\mathcal{L} \mathcal{U}_{1}^{0} \leq-n H^{n-1} \sum_{i=0,2} \gamma_{i}\left(\Phi^{2}-2 \bar{p}_{i} \Phi\right)+\mathcal{E}_{2},
$$

where the error term $\mathcal{E}_{2}$ satisfies the bound $\left|\mathcal{E}_{2}\right| \lesssim H^{n+\frac{3}{2 k}-2+\delta}+C \mathcal{U}_{0}^{N}$ as before ${ }^{1}$.

\footnotetext{
${ }^{1}$ The interested reader can check that expanding the square around $p_{i}$ instead of $\bar{p}_{i}$ would lead to troublesome terms.
} 
Since these terms oscillate very rapidly, we would like to replace them by their averaged effect over one period of the middle oscillator. To leading order, the terms $p_{i} \Phi$ and $\Phi^{2}$ will average out to 0 and $\kappa_{k} E_{1}^{\frac{2}{k}-1}$ respectively. The latter contribution will turn out to be the dominant term leading to an overall dissipation of energy. Defining $\Psi$ and $\Phi^{(2)}$ as in (5.6) and (4.5) respectively, we finally set

$$
\mathcal{U}_{1}=\mathcal{U}_{1}^{0}+n H^{n-1} \sum_{i=0,2} \gamma_{i}\left(\Psi-2 \bar{p}_{i} \Phi^{(2)}\right) .
$$

Our investigation of $\mathcal{L} \mathcal{U}_{1}$ begins with

$$
\begin{aligned}
\mathcal{L} H^{n-1} \Psi=- & H^{n-1}\left(\kappa_{k} E_{1}^{\frac{2}{k}-1}-\Phi^{2}\right)+H^{n-1}\left(\mathcal{R}^{\prime}\left(E_{1}\right)+\left(q_{0}+q_{2}-2 q_{1}\right) \partial_{P} \Psi\right) \\
& +(n-1) H^{n-3} \Psi \sum_{i=0,2}\left(H\left(\frac{\sigma_{i}^{2}}{2}-\gamma_{i} p_{i}^{2}\right)+\frac{1}{2}(n-2) \sigma_{i}^{2} p_{i}^{2}\right) \\
=- & H^{n-1}\left(\kappa_{k} E_{1}^{\frac{2}{k}-1}-\Phi^{2}\right)+I_{9}+I_{10} .
\end{aligned}
$$

Since the function $\mathcal{R}^{\prime}$ has compact support, we have for example $\left|\mathcal{R}^{\prime}\left(E_{1}\right)\right| \lesssim E_{1}^{-1}$. Using the trick from Lemma 4.3 , this yields $\left|\mathcal{R}^{\prime}\left(E_{1}\right)\right| \lesssim H^{-1} \mathcal{U}_{0}$. This allows us to obtain the bound

$$
\left|I_{9}\right| \lesssim H^{n-2} \mathcal{U}_{0}+H^{n-1+\frac{1}{2 k}} E_{1}^{\frac{5}{2 k}-2} \lesssim H^{n-2} \mathcal{U}_{0}+H^{n-3+\frac{3}{k}} \mathcal{U}_{0}^{2-\frac{5}{2 k}}
$$

Since $\Psi$ is bounded, one furthermore has

$$
\left|I_{10}\right| \lesssim H^{n-2} \mathcal{U}_{0}+H^{n-3} \mathcal{U}_{0} .
$$

Turning to the second term in $\mathcal{L U}_{1}$, we have

$$
\begin{aligned}
\mathcal{L} H^{n-1} \bar{p}_{i} \Phi^{(2)}= & H^{n-1} \bar{p}_{i} \Phi+H^{n-1} \bar{p}_{i}\left(q_{0}+q_{2}-2 q_{1}\right) \partial_{P} \Phi^{(2)} \\
& +(n-1) H^{n-3} \bar{p}_{i} \Phi^{(2)} \sum_{j=0,2}\left(H\left(\frac{\sigma_{j}^{2}}{2}-\gamma_{j} p_{j}^{2}\right)+\frac{1}{2}(n-2) \sigma_{j}^{2} p_{j}^{2}\right) \\
& +H^{n-1} \Phi^{(2)}\left(-\bar{q}_{i}\left|\bar{q}_{i}\right|^{2 k-2}-\bar{q}_{i}+R_{p}^{i}-\gamma_{i} \bar{p}_{i}\right)+(n-1) H^{n-2} \sigma_{i}^{2} p_{i} \Phi^{(2)} \\
= & H^{n-1} \bar{p}_{i} \Phi+I_{11}+I_{12}+I_{13}+I_{14} .
\end{aligned}
$$

Using the bound on $R_{p}^{i}$ from (4.12), the error terms appearing in this expression can be bounded by

$$
\begin{aligned}
& \left|I_{11}\right| \lesssim H^{n-1+\frac{1}{2 k}} \mathcal{U}_{0}^{\frac{1}{2}} E_{1}^{\frac{3}{2 k}-\frac{3}{2}} \lesssim H^{n-\frac{5}{2}+\frac{2}{k}} \mathcal{U}_{0}^{2-\frac{3}{2 k}} \\
& \left|I_{12}\right| \lesssim H^{n-2} \mathcal{U}_{0}^{\frac{3}{2}}+H^{n-3} \mathcal{U}_{0}^{\frac{3}{2}}, \\
& \left|I_{13}\right| \lesssim H^{n-1} E_{1}^{\frac{3}{2 k}-1}\left(\mathcal{U}_{0}+\mathcal{U}_{0}^{2} H^{\frac{3}{2 k}-1}\right) \lesssim H^{n-2+\frac{3}{2 k}} \mathcal{U}_{0}^{3-\frac{3}{2 k}}, \\
& \left|I_{14}\right| \lesssim H^{n-2} \mathcal{U}_{0}^{\frac{1}{2}}
\end{aligned}
$$

By Young's inequality, it can be checked that, for every $\delta>0$ there exist constants $C$ and $N$ such that $\left|I_{j}\right| \leq H^{n-2+\frac{3}{2 k}+\delta}+C \mathcal{U}_{0}^{N}$ for $j=9, \ldots, 14$. Inserting these bounds into (5.8) and (5.9), this shows that

$$
\mathcal{L} \mathcal{U}_{1} \leq-n \kappa_{k}\left(\gamma_{0}+\gamma_{2}\right) H^{n-1} E_{1}^{\frac{2}{k}-1}+H^{n-2+\frac{3}{2 k}+\delta}+C \mathcal{U}_{0}^{N} .
$$


Since $\frac{2}{k}-1 \leq 0$ and since $n-2+\frac{2}{k}>n-2+\frac{3}{2 k}+\delta$ for sufficiently small $\delta$, we finally obtain

$$
\mathcal{L} \mathcal{U}_{1} \leq-\frac{1}{2} n \kappa_{k}\left(\gamma_{0}+\gamma_{2}\right) H^{n-2+\frac{2}{k}}+C \mathcal{U}_{0}^{N}
$$

for a possibly different constant $C$. This is precisely the bound (5.2) which was the missing piece to complete the proof of Theorem 5.6, the principal result of this article.

\section{References}

[BP78] G. BLANKEnShip and G. C. PAPAnicolaou. Stability and control of stochastic systems with wide-band noise disturbances. I. SIAM J. Appl. Math. 34, no. 3, (1978), 437-476.

[BK07] J. BRICMONT and A. KUPIAINEN. Towards a derivation of Fourier's law for coupled anharmonic oscillators. Comm. Math. Phys. 274, no. 3, (2007), 555-626.

[BLP78] A. Bensoussan, J.-L. Lions, and G. PAPAnicolaou. Asymptotic analysis for periodic structures, vol. 5 of Studies in Mathematics and its Applications. NorthHolland Publishing Co., Amsterdam, 1978.

[BLR00] F. Bonetto, J. L. Lebowitz, and L. Rey-Bellet. Fourier's law: a challenge to theorists. In Mathematical physics 2000, 128-150. Imp. Coll. Press, London, 2000.

[DFG06] R. Douc, G. ForT, and A. Guillin. Subgeometric rates of convergence of $f$ ergodic strong markov processes, 2006. Preprint.

[EE87] D. E. Edmunds and W. D. Evans. Spectral theory and differential operators. Oxford Mathematical Monographs. The Clarendon Press Oxford University Press, New York, 1987. , Oxford Science Publications.

[EH00] J.-P. ECKMANN and M. HAIRER. Non-equilibrium statistical mechanics of strongly anharmonic chains of oscillators. Comm. Math. Phys. 212, no. 1, (2000), 105-164.

[EH03] J.-P. ECKMANN and M. HAIRER. Spectral properties of hypoelliptic operators. Comm. Math. Phys. 235, no. 2, (2003), 233-253.

[EPR99a] J.-P. Eckmann, C.-A. Pillet, and L. Rey-Bellet. Entropy production in nonlinear, thermally driven Hamiltonian systems. J. Statist. Phys. 95, no. 1-2, (1999), 305-331.

[EPR99b] J.-P. Eckmann, C.-A. Pillet, and L. Rey-Bellet. Non-equilibrium statistical mechanics of anharmonic chains coupled to two heat baths at different temperatures. Comm. Math. Phys. 201, no. 3, (1999), 657-697.

[GW69] K. Gustafson and J. Weidmann. On the essential spectrum. J. Math. Anal. Appl. 25, (1969), 121-127.

[Has80] R. Z. HAS'MINSKII. Stochastic stability of differential equations, vol. 7 of Monographs and Textbooks on Mechanics of Solids and Fluids: Mechanics and Analysis. Sijthoff \& Noordhoff, Alphen aan den Rijn, 1980. Translated from the Russian by D. Louvish.

[HLW06] E. HAIRER, C. LUBICH, and G. WANNER. Geometric numerical integration, vol. 31 of Springer Series in Computational Mathematics. Springer-Verlag, Berlin, second ed., 2006. Structure-preserving algorithms for ordinary differential equations.

[HN04] F. HÉRAU and F. NIER. Isotropic hypoellipticity and trend to equilibrium for the Fokker-Planck equation with a high-degree potential. Arch. Ration. Mech. Anal. 171, no. 2, (2004), 151-218.

[HN05] B. HELFFER and F. NIER. Hypoelliptic estimates and spectral theory for FokkerPlanck operators and Witten Laplacians, vol. 1862 of Lecture Notes in Mathematics. Springer-Verlag, Berlin, 2005. 
[Kat95] T. KATO. Perturbation theory for linear operators. Classics in Mathematics. Springer-Verlag, Berlin, 1995. Reprint of the 1980 edition.

[KB37] N. Kryloff and N. BogolioubofF. La théorie générale de la mesure dans son application à l'étude des systèmes dynamiques de la mécanique non linéaire. Ann. of Math. (2) 38, no. 1, (1937), 65-113.

[LLP03] S. LEPRI, R. LiVI, and A. POLITI. Thermal conduction in classical low-dimensional lattices. Phys. Rep. 377, no. 1, (2003), 1-80.

[LS05] R. LefEVERE and A. Schenkel. Normal heat conductivity in a strongly pinned chain of anharmonic oscillators, 2005. URL arXiv: cond-mat/0507560v4.

[MA94] R. S. MACKAY and S. AUBRY. Proof of existence of breathers for time-reversible or Hamiltonian networks of weakly coupled oscillators. Nonlinearity 7, no. 6, (1994), $1623-1643$.

[Nie06] F. NIER. Hypoellipticity for Fokker-Planck operators and Witten Laplacians, 2006. Preprint.

[RBT02] L. REY-Bellet and L. E. Thomas. Exponential convergence to non-equilibrium stationary states in classical statistical mechanics. Comm. Math. Phys. 225, no. 2, (2002), 305-329.

[RLL67] Z. Rieder, J. L. Lebowitz, and E. Lieb. Properties of a harmonic crystal in a stationary nonequilibium state. J. Math. Phys. 8, no. 5, (1967), 1073-1078.

[RS78] M. REED and B. Simon. Methods of modern mathematical physics. IV. Analysis of operators. Academic Press [Harcourt Brace Jovanovich Publishers], New York, 1978.

[Ver97] A. Y. Veretennikov. On polynomial mixing bounds for stochastic differential equations. Stochastic Process. Appl. 70, no. 1, (1997), 115-127.

[Vil07] C. Villani. Hypocoercivity, 2007. To appear in Memoirs of the AMS.

[Wol59] F. Wolf. On the essential spectrum of partial differential boundary problems. Comm. Pure Appl. Math. 12, (1959), 211-228. 